\title{
Long-term patterns of an interconnected core marine microbiota
}

Anders K. Krabberød ${ }^{1 *}$, Ina M. Deutschmann², Marit F. M. Bjorbækmo ${ }^{1}$, Vanessa Balagué $^{2}$, Caterina R. Giner ${ }^{2}$, Isabel Ferrera ${ }^{2,3}$, Esther Garcés ${ }^{2}$, Ramon Massana ${ }^{2}$, Josep M. Gasol ${ }^{2,4}$, Ramiro Logares ${ }^{2,1^{*}}$

${ }^{1}$ University of Oslo, Department of Biosciences, Section for Genetics and Evolutionary Biology (Evogene), Blindernv. 31, N-0316 Oslo, Norway

${ }^{2}$ Institute of Marine Sciences (ICM), CSIC, Passeig Marítim de la Barceloneta, Barcelona, Spain

3 Centro Oceanográfico de Málaga, Instituto Español de Oceanografía, 29640 Fuengirola, Málaga, Spain

${ }^{4}$ Centre for Marine Ecosystems Research, School of Sciences, Edith Cowan University, Joondalup, WA, Australia

\section{Corresponding authors:}

*Anders K. Krabberød, University of Oslo, Department of Biosciences, Section for Genetics and Evolutionary Biology (Evogene), Blindernv. 31, N-0316 Oslo, Norway Email: a.k.krabberod@ibv.uio.no

*Ramiro Logares, Institute of Marine Sciences (ICM), CSIC, Passeig Marítim de la Barceloneta, Barcelona, Spain

Email: ramiro.logares@icm.csic.es

Running title: Patterns in a core marine microbiota

Manuscript for: Microbiome 


\section{ABSTRACT}

42

\section{Background}

Ocean microbes constitute $\sim 70 \%$ of the marine biomass, are responsible for $\sim 50 \%$ of the Earth's primary production, and are crucial for global biogeochemical cycles. Marine microbiotas include core taxa that are usually key for ecosystem function. Despite their importance, core marine microbes are relatively unknown, which reflects the lack of consensus on how to identify them. So far, most core microbiotas have been defined based on species occurrence and abundance. Yet, species interactions are also important to identify core microbes, as communities include interacting species. Here, we investigate interconnected bacteria and small protists of the core pelagic microbiota populating a long-term marine-coastal observatory in the Mediterranean Sea over a decade.

\section{Results}

Core microbes were defined as those present in $>30 \%$ of the monthly samples over 10 years, with the strongest associations. The core microbiota included 259 Operational Taxonomic Units (OTUs) including 182 bacteria, 77 protists, and 1,411 strong and mostly positive $(\sim 95 \%)$ associations. Core bacteria tended to be associated with other bacteria, while core protists tended to be associated with bacteria. The richness and abundance of core OTUs varied annually, decreasing in stratified warmers waters and increasing in colder mixed waters. Most core OTUs had a preference for one season, mostly winter, which featured subnetworks with the highest connectivity. Groups of highly associated taxa tended to include protists and bacteria with predominance in the same season, particularly winter. A group of 13 highly-connected hub-OTUs, with potentially important ecological roles dominated in winter and spring. Similarly, 18 connector OTUs with a low degree but high centrality were mostly associated with summer or autumn and may represent transitions between seasonal communities.

\section{Conclusions}

We found a relatively small and dynamic interconnected core microbiota in a model temperate marine-coastal site, with potential interactions being more deterministic in winter than in other seasons. These core microbes would be essential for the functioning of this ecosystem over the year. Other non-core taxa may also carry out important functions but would be redundant and non-essential. Our work contributes to the understanding of the dynamics and potential interactions of core microbes possibly sustaining ocean ecosystem function.

Keywords: bacteria, protists, ocean, time-series, seasonality, networks, associations 


\section{BACKGROUND}

83 Ecosystems are composed of interacting units embedded in and influenced by their physicochemical environment. Ecosystem function can be broadly defined as the the ecosystem towards a new state or its collapse. It is hypothesized that ecological redundancy guarantees continuous ecosystem function, as multiple species could carry out the same or similar function [1]. And while the amount of functional redundancy in microbial ecosystems is a matter of debate $[2,3]$ it has also been observed that microbiotas in comparable habitats tend to share "core" species that are hypothesized to be fundamental for ecosystem function [4]. These core organisms and the functions they carry out might not be easily replaced.

Identifying the core microbiota is not straightforward as there are different ways spatiotemporal scales. This definition might not be sufficient, however, since communities are made up of interacting species [5]. A more appropriate definition of a core, therefore, needs to incorporate ecological interactions fundamental for the community in the location under study $[4,5]$. This is particularly important in studies

101 using DNA to investigate microbial communities, as a fraction of the detected taxa 102 could be dormant, dead, or transient [6-8]. In the interaction-based definition taxa that 103 do not appear to be interacting are excluded from the core [4]. Core microbiotas based on common presence have been widely studied in terrestrial animals, in particular humans [9] or cattle [10], as well in marine animals, in 
systems, such as soils or the ocean, have been investigated to a lesser extent. In soils,

108 for example, a global analysis identified a core group of 241 ubiquitous and dominant

109 bacterial taxa with more or less invariant abundances and unclear habitat preferences

110 [15]. In the tropical and subtropical global-ocean, a total of 68 bacteria and 57

111 picoeukaryotic operational taxonomic units (OTUs) have been identified that could be 112 part of the core surface microbiota, as they were present in $>80 \%$ of the globally113 distributed samples [16].

114 Analyses of ocean time-series have also pointed to the existence of core 115 microbiotas. For example, Gilbert et al. [17] investigated the microbiota of the English 116 Channel for 6 years and found 12 abundant OTUs that were detected throughout the 117 entire dataset (72 time-points), totaling $\sim 35 \%$ of the sequence abundance. Potentially 118 core bacterial OTUs were detected in the SPOT time-series (southern California), in a 119 study covering 10 years of monthly samples in the euphotic zone [18]. These 120 potentially-core bacterial OTUs were present in $>75 \%$ of the months, represented $\sim 7 \%$ 121 (25-28 OTUs depending on depth) of the total richness, and had a high $(>10 \%)$ relative 122 abundance [18].

123 These studies have provided substantial insights on core marine microbiotas, 124 although they typically define them in terms of species occurrence or abundance over 125 spatiotemporal scales, rather than on potential interactions. As in other ecosystems, 126 microbial interactions are essential for the functioning of the ocean ecosystem, where 127 they guarantee the transfer of carbon and energy to upper trophic levels, as well as the 128 recycling of carbon and nutrients [19]. Despite their importance, most microbial 129 interactions in the ocean remain unknown [20]. A recent literature survey spanning the 130 last 150 years indicated that we have documented a minor fraction of protist interactions 131 in the ocean [21] and most likely, the same is true if not worse for bacteria. 
133 knowledge gap. Association networks are based on correlations between species'

134 abundances and they may reflect microbial interactions [22]. Contemporaneous

135 positive correlations may point to interactions such as symbiosis, or similar niche

136 preferences, while negative correlations may suggest predation, competition, or

137 opposite niche preferences [23]. So far, network analyses have produced hypotheses on

138 microbial interactions at the level of individual species across diverse ecosystems [22,

$13924,25]$, a few of which have been experimentally validated [26]. In addition, networks

140 can help detect species that have relatively more associations to other species ("hubs"),

141 or species that connect different subgroups within a network, and which therefore may

142 have important roles in the ecosystem. Groups of highly associated species in the 143 network ("modules") may represent niches [27, 28], and the amount of these modules

144 may increase with increasing environmental selection [22]. Networks can also produce 145 ecological insight at the community level, since their architecture can reflect 146 community processes, such as selection [27].

147 Network analyses have been particularly useful for the investigation of 148 microbial interactions in the ocean $[25,29]$. A surface global-ocean network analysis 149 of prokaryotes and single-celled eukaryotes indicated that $\sim 72 \%$ of the associations 150 between microbes were positive and that most associations were between single-celled 151 eukaryotes belonging to different organismal size-fractions [26]. Other studies using 152 networks have indicated a limited number of associations between marine microbes and 153 abiotic environmental variables [17, 18, 23, 26, 30-32], suggesting that microbial 154 interactions have an important role in driving community turnover [32]. Despite the 155 important insights these studies have provided, most of them share the limitation that 
they do not disentangle whether microbial associations may represent ecological

157 interactions or environmental preferences [22].

Even though association networks based on long-term species dynamics may

159 allow a more accurate delineation of core marine microbiotas, few studies have

160 identified them in this manner. Consequently, we have a limited understanding of the

161 interconnected set of organisms that may be key for ocean ecosystem function. Here

162 we identify and investigate the core microbiota occurring in the marine-coastal Blanes

163 Bay Microbial Observatory (Northwestern Mediterranean Sea) over 10 years. We

164 delineated the core microbiota stringently, using potential interactions based on species

165 abundances. We also made an effort to disentangle environmental effects in association

166 networks by identifying and removing species associations that are a consequence of

167 shared environmental preference and not interactions between the species [33]. We

168 analyzed bacteria and protists from the pico- $(0.2-3 \mu \mathrm{m})$ and nanoplankton $(3-20 \mu \mathrm{m})$

169 organismal size fractions, which show a strong seasonality in this location [34-36].

170 Taxa relative abundances were estimated by sequencing the $16 \mathrm{~S}$ and $18 \mathrm{~S}$ rRNA-gene

171 and delineating OTUs as Amplicon Sequence Variants (ASVs). Specifically, we ask:

172 What taxa constitute the interconnected core microbiota and what are the main patterns

173 of this assemblage over 10 years? Does the core microbiota feature seasonal sub-groups

174 of highly associated species? What degree of association do bacteria and microbial

175 eukaryotes have and do they show comparable connectivity? Can we identify core

176 OTUs with central positions in the network that could have important ecological roles?

\section{RESULTS}


180 Based on the data set containing 2,926 OTUs, (1,561 bacteria and 1,365 microbial

181 eukaryotes) we first defined the resident OTUs as the bacteria and microbial eukaryotes

182 present in $>30 \%$ of the samples, which equals 36 out of 120 months (not necessarily

183 consecutive). This threshold was selected as it includes seasonal OTUs that would be

184 present recurrently in at least one season. The residents consisted of 709 OTUs: 354

185 Bacteria ( $\sim 54 \%$ relative read abundance) and 355 Eukaryotic OTUs ( $\sim 46 \%$ relative

186 read abundance) [Table 1, see methods for calculation of relative read abundance]. The

187 most abundant resident bacteria OTUs belonged to Oxyphotobacteria (mostly

188 Synechococcus; $\sim 15 \%$ of total relative read abundance), Alphaproteobacteria (mostly

189 SAR11 Clade Ia [ 9\%, and clade II [ 4\%]), and Gammaproteobacteria (mainly SAR86;

$190 \sim 2 \%$ ). The most abundant resident protist OTUs belonged to Dinophyceae

191 (predominantly an unclassified dinoflagellate lineage [ 7\%], Syndiniales Group I

192 Clade $1[\sim 7 \%]$ and Gyrodinium [ 4\%]), Chlorophyta (mostly Micromonas $[\sim 3 \%]$ and

193 Bathycoccus [ 2\%]), Ochrophyta (predominantly Mediophyceae $[\sim 2 \%]$ and

194 Chaetoceros $[\sim 1 \%]$ ) and Cryptophyceae (mainly a Cryptomonadales lineage $[\sim 2 \%]$ )

195 [Figure 3, Table S1, Additional file 1].

197 Table 1. Description of the datasets.

\begin{tabular}{|c|c|c|c|}
\hline & OTUs & OTUs (\%) & Sequence abundance $(\%)^{*}$ \\
\hline All OTUs $^{1}$ & 2,926 & 100 & 100 \\
\hline Bacteria & 1,561 & 53.3 & 50.7 \\
\hline Protists & 1,365 & 46.7 & 49.3 \\
\hline Resident microbiota ${ }^{2}$ & 709 & 100 & $100(85)$ \\
\hline Bacteria & 354 & 49.9 & 53.6 \\
\hline Protists & 355 & 50.1 & 46.4 \\
\hline Core microbiota $^{3}$ & 259 & 100 & $64.5(54)$ \\
\hline Bacteria & 182 & 70.3 & 46.3 \\
\hline Protists & 77 & 29.7 & 18.2 \\
\hline Picoplankton & 109 & 42.1 & 32.4 \\
\hline Nanoplankton & 150 & 57.9 & 32.1 \\
\hline \multicolumn{4}{|l|}{ Protists } \\
\hline Heterotroph & 5 & 1.9 & 0.3 \\
\hline Photoautotroph & 37 & 14.3 & 11.8 \\
\hline Parasite & 21 & 8.1 & 3.5 \\
\hline Mixotroph & 3 & 1.2 & 0.7 \\
\hline
\end{tabular}




\begin{tabular}{lccc}
\hline Symbiont & 1 & 0.4 & 0.1 \\
Unknown & 11 & 4.3 & 2.0 \\
Bacteria & & & 19.3 \\
Photoautotroph (cyanobacteria) & 19 & 7.3 & 26.8 \\
Non-photoautotroph & & 62.5 & 21.8 \\
Seasonal preference core OTUs & 163 & 60.2 & 16.4 \\
Winter & 156 & 9.3 & 8.2 \\
Spring & 24 & 17.0 & 13.7 \\
Summer & 44 & 11.6 & 4.5 \\
Autumn & 30 & 1.9 & 21.8 \\
No seasonality & 5 & & 13.7 \\
Seasonal subnetworks & 156 & 60.2 & 6.6 \\
Winter & 19 & 7.3 & 12.9 \\
Spring & 41 & 15.8 & \\
Summer & 26 & 10.0 & \\
Autumn & & & \\
\hline
\end{tabular}

${ }_{1}^{1}$ Number of OTUs in the full dataset that were left after quality control and rarefaction, which were present in at least $10 \%$ of the samples (i.e. 12 months, not necessarily consecutive).

2 OTUs present in at least $30 \%$ of the samples (i.e. 36 months, not necessarily consecutive) [=Resident microbiota].

3 OTUs included in the core network (core microbiota) with significant correlations ( $p \& q<0.001$ ), local similarity scores $>|0.7|$ and Spearman correlations $>|0.7|$, being present in at least $30 \%$ of the samples.

${ }^{4}$ Includes non-photoautotrophic lifestyles (i.e., chemoautotrophs, photoheterotrophs, chemoheterotrophs, etc.).

* In Italics the abundances relative to all OTUs are indicated. All other values in normal text indicate abundances relative to OTUs in the resident microbiota.

The resident microbiota, including both protists and bacteria, showed seasonal

variation over 10 years, with communities from the same season but different years

tending to group (Figure 1C and D). The structure of the resident microbiota correlated

to specific environmental variables during winter (nutrients, Total photosynthetic

a [Chla]), summer (daylength, temperature, Secchi disk depth and, the cell abundances

217 1D], leading to a dbRDA constrained and unconstrained variation of $41 \%$ and $59 \%$

218 respectively (Figure 1D). The selected variables were predominantly aligned with the 219 axis summer (daylength, temperature, and the cell abundance of Synechococcus and $220 \mathrm{HP})$ - winter $\left(\mathrm{SiO}_{2}\right.$, small PNF [Figure 1D]. This dbRDA axis had the highest 221 eigenvalue, explaining $\sim 55 \%$ of the constrained variation (Figure 1D). Even though 222 the measured environmental variables did not explain the majority of the variation of 
223 the resident microbiota, they could account for a substantial fraction. This was further

224 supported by Adonis analyses, which indicated that the measured environmental

225 variables could explain $\sim 45 \%$ of the resident microbiota variance, with temperature and

226 daylength having a predominant role by accounting for $30 \%$ of this variance $(15 \%$

227 each).

228 We then investigated whether temperature and daylength could determine the

229 main niches. We found that $\sim 70 \%$ and $\sim 68 \%$ of the OTUs in the resident microbiota

230 had niche preferences associated with temperature or daylength respectively (Figure

231 1E-F; Note that several OTUs preferring Spring or Autumn are not expected to be

232 detected with this approach, as their preferred temperature or daylength may not differ

233 significantly from the randomized mean). In total, 371 OTUs from the resident

234 microbiota had both a temperature and a daylength niche preference that departed

235 significantly from the randomization mean (Figure 1E-F). These 371 OTUs

236 represented $\sim 52 \%$ of all OTUs in the resident microbiota, corresponding to $\sim 90 \%$ of

237 the sequence abundance. In particular, 248 OTUs had a weighted mean for both

238 temperature and daylength below the randomization mean (corresponding to

239 winter/autumn), while 116 OTUs had a weighted mean above the randomization mean

240 for both variables (corresponding to summer/spring). Interestingly, 7 OTUs displayed

241 a weighted mean above and below the randomized mean for temperature and daylength

242 respectively (corresponding to autumn or spring). 


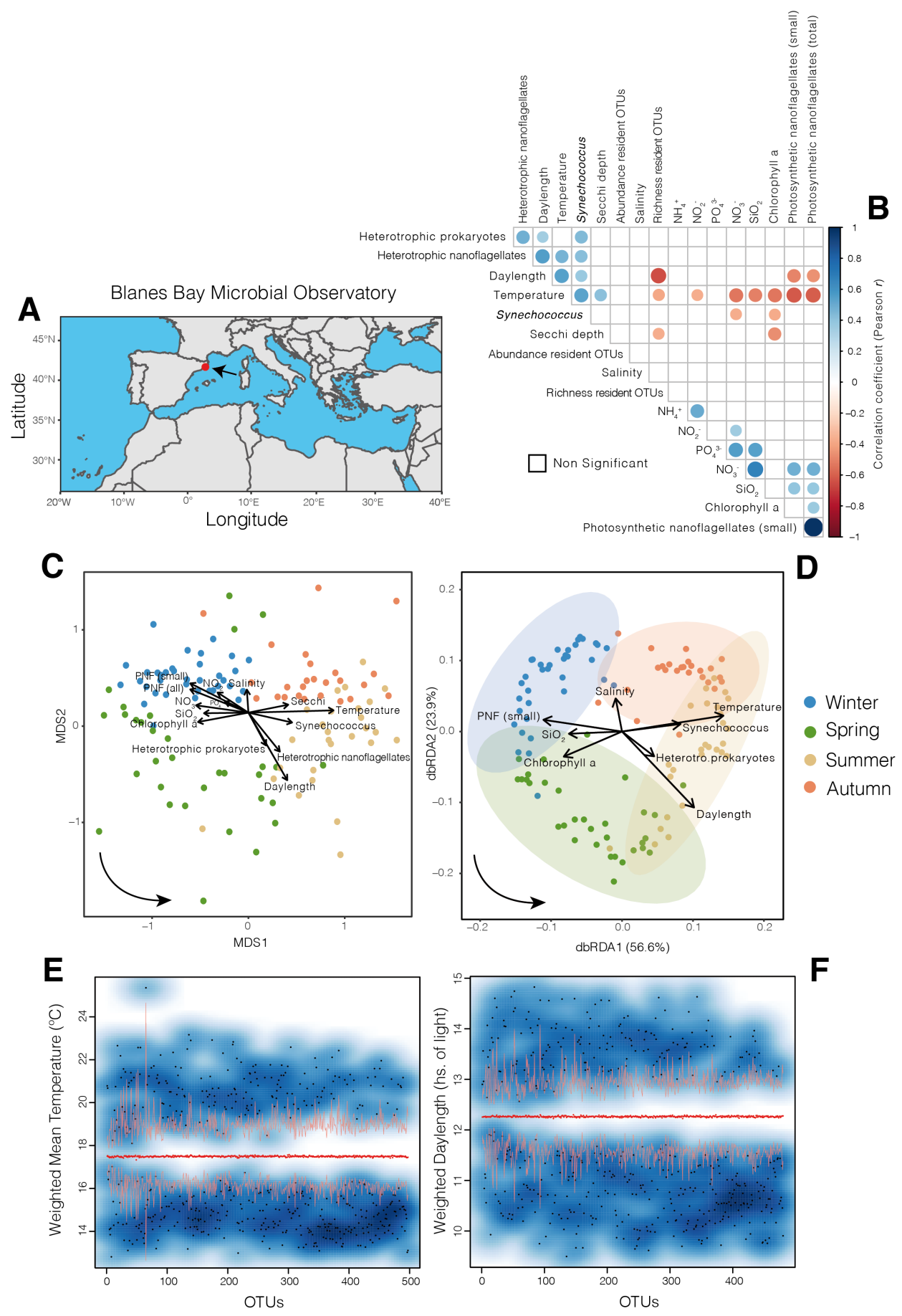

244 Figure 1. The Blanes Bay Microbial Observatory and the variation of its resident

245 microbiota and measured environmental variables over ten years. A) Location of the Blanes

246 Bay Microbial Observatory. B) All possible correlations between the measured environmental

247 variables including the richness and abundance of resident OTUs (NB: only 709 resident OTUs

248 are considered, see Table1). Only significant Pearson correlation coefficients are shown

249 ( $p<0.01)$. The $p$-values were corrected for multiple inference (Holm's method). C) Unconstrained 
251 only, to which environmental variables were fitted. Only variables with a significant fit are shown

$252(P<0.05)$. Arrows indicate the direction of the gradient and their length represents the strength

253 of the correlation between resident OTUs and a particular environmental variable. The color of

254 the samples (circles) indicates the season to which they belong. The bottom-left arrow indicates

255 the direction of the seasonal change. PNF = photosynthetic nanoflagellates. D) Constrained

256 ordination (Distance-based redundancy analyses, dbRDA, using Bray Curtis dissimilarities)

257 including only the most relevant variables after stepwise model selection using permutation tests.

258 Each axis (i.e., dbRDA1 and dbRDA2) indicates the amount of variance it explains according to

259 the associated eigenvalues. The color of the samples (circles) indicates the season to which they

260 belong. Arrows indicate the direction of the gradient and their length represents the strength of

261 the correlation between resident OTUs and a particular environmental variable. The bottom-left

262 arrow indicates the direction of the seasonal change. E-F) Resident OTUs displaying different

263 niche preferences (blueish areas) in terms of the two most important abiotic variables:

264 Temperature E) and Daylength F). The red dots indicate the randomization mean, and the orange

265 curves represent the confidence limits. Black dots indicate individual OTUs for which temperature

266 or daylength preferences are significantly $(p<0.05)$ higher or lower than a random distribution

267 over 10 years. At least two assemblages with different niches become evident: one preferring

268 higher temperature and longer days (summer/spring), and another one preferring lower

269 temperature and shorter days (winter/autumn). Note that several OTUs associated to Spring or

270 Autumn are not expected to be detected with this approach, as their preferred temperature or

271 daylength may not differ significantly from the randomized mean. 
274 To determine the core microbiota that incorporates possible interactions, we

275 constructed an association network based on the resident OTUs and removed all OTUs

276 that were not involved in strong and significant associations with any other OTUs.

277 Specifically, we kept only the associations (edges in the network) with Local similarity

278 score $|\mathrm{LS}|>0.7$, a false discovery rate adjusted $\mathrm{p}$-value $<0.001$ and Spearman $|\mathrm{r}|>0.7$.

279 In addition, we removed all associations that seemed to be caused by environmental 280 preferences of OTUs (see Methods). The core network consisted of 1,411 significant 281 and strong correlations (Figure 2A) and was substantially smaller than the network 282 based on the resident OTUs without stringent cut-offs (Figure S1A, Additional file 2, 283 removed edges in Figure S1B, Additional file 2). The core network includes only the 284 strongest microbial associations that are inferred during a decade and, according to our 285 definition, determines the core microbiota. The associations in the core microbiota may 286 represent proxies for species interactions since steps have been taken to remove 287 associations that are driven by environmental factors.

288 In the core network, most associations were positive $(\sim 95 \%)$, pointing to the 289 dominance of co-existence or symbiotic associations (Table 2, Figure 2A). The core 290 network had "small world" properties [37], with a small average path length (i.e. 291 number of nodes between any pair of nodes through the shortest path) and a relatively 292 high clustering coefficient, showing that nodes tend to be connected to other nodes, 293 forming tightly knit groups, more than what it would be expected by chance (Table 3). 294 Since node degree was not correlated with OTU abundance (Figure S2, Additional file 295 3), the associations between OTUs are not caused by a high sequence abundance alone, 296 as the most abundant OTUs did not tend to be the most connected. 
bioRxiv preprint doi: https://doi.org/10.1101/2021.03.18.435965; this version posted June 24, 2021. The copyright holder for this preprint (which was not certified by peer review) is the author/funder, who has granted bioRxiv a license to display the preprint in perpetuity. It is made available under aCC-BY-NC-ND 4.0 International license.
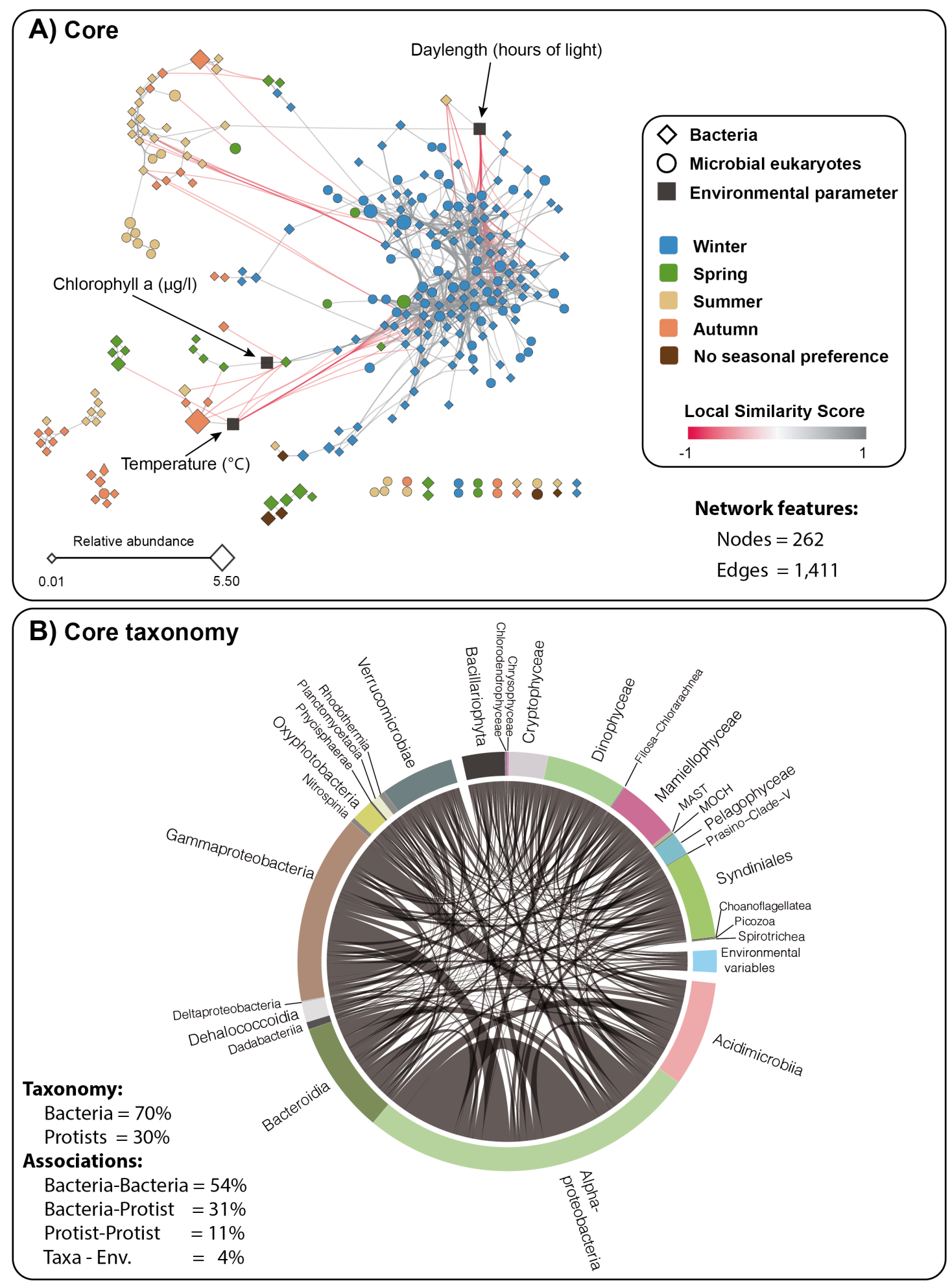

299 Figure 2. Core microbiota resulting from 10 years of monthly pico- and nanoplankton

300 relative abundances. A) Core network including bacteria and microbial eukaryotic OTUs that

301 occur $\geq 30 \%$ of the time during the studied decade (i.e. resident microbiota), with highly 

removed. The color of the edges (links) indicates whether the association is positive (grey) or negative (red). The shape of nodes indicates bacteria (rhomboid) or microbial eukaryotes (circle), and the color of nodes represents species seasonal preferences, determined using the indicator value (indval, $p<0.05$ ). Node size indicates OTU relative abundance. B) Core network as a Circos plot, indicating the high-rank taxonomy of the core OTUs. Since $95 \%$ of the associations are positive (see Table 2), we do not indicate whether an edge is positive or negative. defined for the other seasons (Figure 2A). Of the 15 environmental variables analyzed, only 3 were found to be significantly correlated with core OTUs: daylength, showing

314 strong correlations with 33 OTUs, temperature, correlated with 14 OTUs, and 315 Chlorophyll a, correlated with 1 OTU (Figure 2A). Therefore, the analysis of the core 316 network also points to the importance of temperature and daylength in the decade-long 317 seasonal dynamics of the studied microbial ecosystem. It is also coherent with the 318 Adonis and ordination analyses (Figure 1C-B). However, the associations between 319 these environmental parameters with taxa represented only $4 \%$ of all the associations 320 (Figure 2B).

321 Table 2. Core associations. See Figure 2.

\begin{tabular}{lccr}
\hline & $\begin{array}{c}\text { Association \# } \\
\text { (edges) }\end{array}$ & $\begin{array}{c}\text { Co-occurrences } \\
\text { (positive) }\end{array}$ & $\begin{array}{c}\text { Co-exclusions } \\
\text { (negative) }\end{array}$ \\
\hline All & 1,411 & $1,341(95.0 \%)$ & $70(5.0 \%)$ \\
Within Picoplankton & 378 & $353(93.3 \%)$ & $25(6.6 \%)$ \\
Within Nanoplankton & 791 & $748(94.6 \%)$ & $43(5.4 \%)$ \\
Picoplankton-Nanoplankton & 242 & $240(99.2 \%)$ & $2(0.8 \%)$ \\
\hline
\end{tabular}

Of the 709 OTUs from the resident microbiota (Figure 3), only 259 OTUs $325(35 \%)$ were left in the core network (182 bacteria $(\sim 70 \%)$ and 77 microbial eukaryotic 
bioRxiv preprint doi: https://doi.org/10.1101/2021.03.18.435965; this version posted June 24, 2021. The copyright holder for this preprint

(which was not certified by peer review) is the author/funder, who has granted bioRxiv a license to display the preprint in perpetuity. It is made available under aCC-BY-NC-ND 4.0 International license.

OTUs ( 30\%); Table 1, Figure 2). The monthly taxonomic composition of the resident

327 microbiota differed from that of the core (Figure 3). The core OTUs accounted for

$328 \sim 64 \%$ of the relative read abundance of the resident microbiota (Table 1). The core

329 OTUs had annual variation in terms of richness and abundance over the 10 years for

330 both the pico- and nanoplankton, with microbial eukaryotes decreasing markedly in

331 OTU richness and relative read abundance in the warmer seasons, and increasing during

332 colder periods (Figure 3).

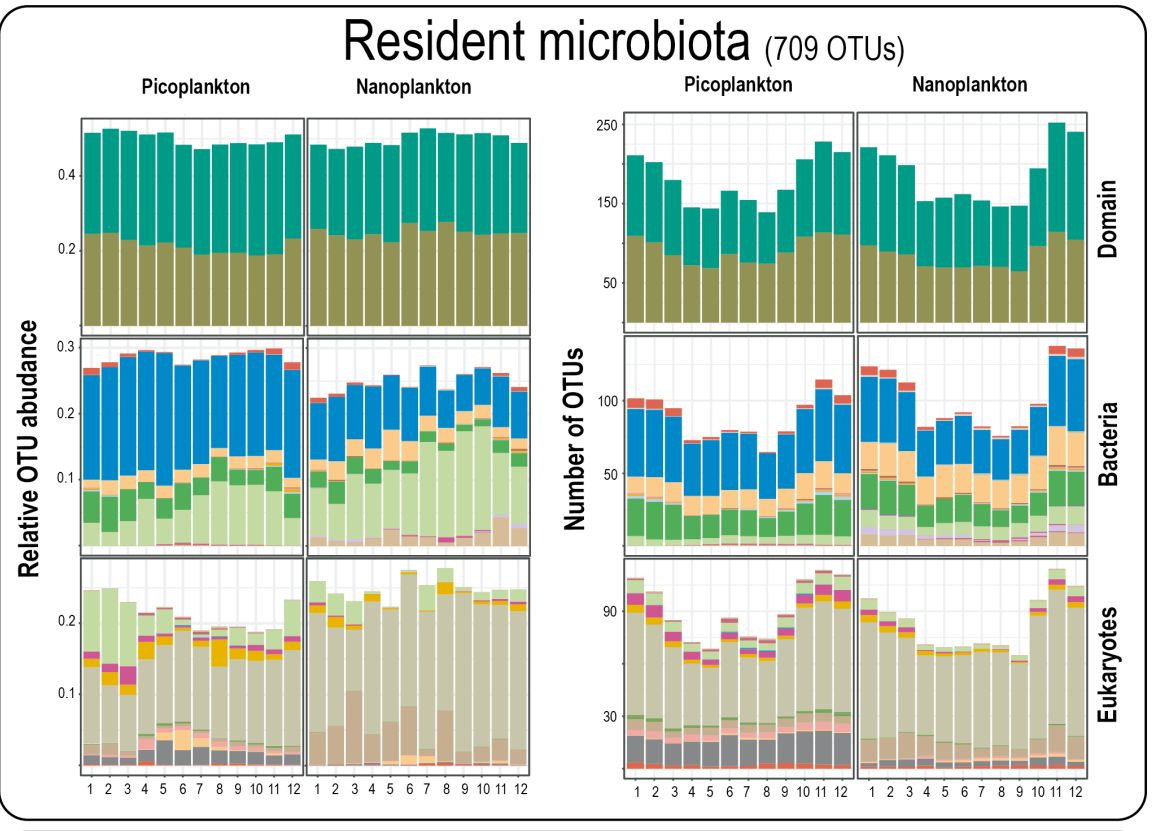

\section{Domain \\ - Bacteria
Eukaryota}

Bacterial Class

Acidimicrobiia

Actinobacteria

Bacilli

Bacteroidia

Campylobacteria

Dehalococcoidia

Deltaproteobacteria

Gammaproteobacteria

Nitrospinia

Oxyphotobacteria

Phycisphaerae

Rhodothermia

Verrucomicrobiae

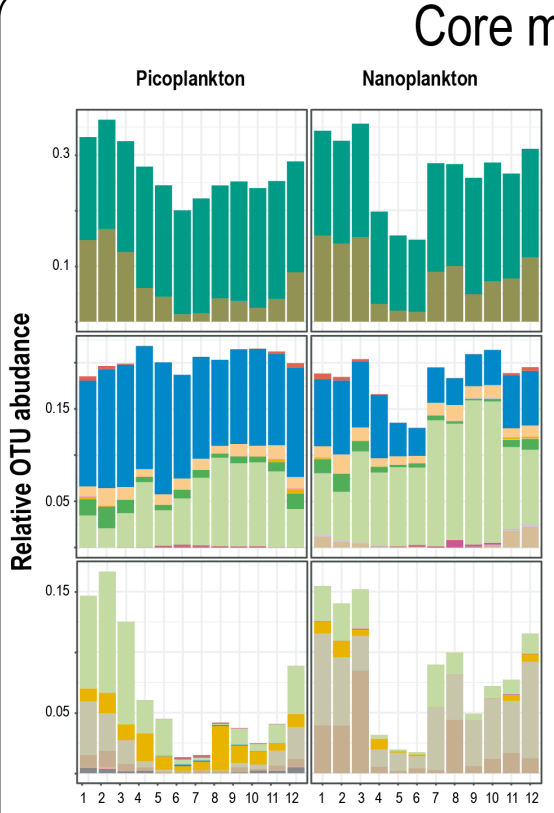

microbiota (259 OTUs)

Nanoplankton

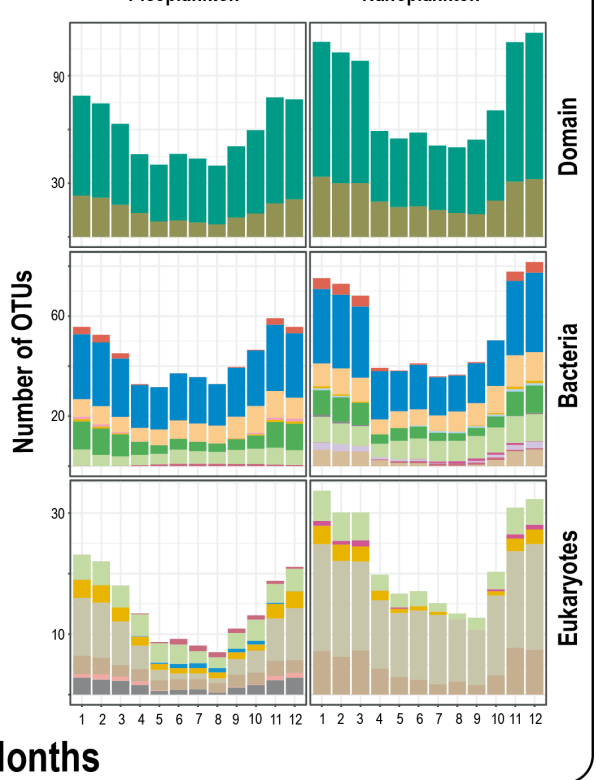

Eukaryotic Phylum

Centroheliozo

- Chlorophyt

Choanoflagellida

Ciliophora

Cryptophyta

Kinoflagellata

Stramenopiles: Ochrophyta

Picozoa
Radiolaria

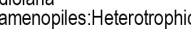

Telonemia 
Figure 3. The monthly variation in the resident and core microbiotas over 10 years. Upper panels: The resident microbiota is defined as those eukaryotes and bacteria that occur in at least $30 \%$ of the samples over 10 years. The relative OTU abundance (left panel) and number of OTUs (right panel) for different domains and taxonomic levels in the resident microbiota are shown. Note that the relative abundance of Bacteria vs. Eukaryotes does not necessarily reflect organismal abundances on the sampling site, but the amplicon relative abundance after PCR.

341 Relative abundances were calculated for each year and aggregated over the corresponding months along the 10 years for the resident microbiota, then split into size fractions (NB: relative

343 abundance for both domains and size fraction sums up to 1 for each month across ten years).

344 Lower panels: Core microbiota over 10 years. The relative abundances of core OTUs reflect the 345 remaining proportions after removing all the OTUs that were not strongly associated when 346 building networks. Relative OTU abundance (left panel) and number of OTUs (right panel) for 347 different domains and taxonomic levels among the core OTUs. core OTUs were Oxyphotobacteria (mostly Synechococcus), total abundance $\sim 14 \%$ of

351 the resident microbiota, followed by Alphaproteobacteria, with SAR11 clades Ia and II representing $\sim 9 \%$ and $\sim 2 \%$ respectively. The most abundant microbial eukaryotic 353 groups were Micromonas, Bathycoccus, Dinophyceae, and Cryptomonadales (each $354 \sim 2 \%$ ) [Figure 3; Table S3, Additional file 1]. In terms of diversity and abundance, 355 bacterial non-phototrophs (including chemoautotrophs, photoheterotrophs, 356 chemoheterotrophs) were the most prevalent in the core microbiota, representing $\sim 62 \%$ 357 of the OTUs and a quarter of the total relative read abundance (Table 1). In turn, 358 protistan heterotrophs represented a minor fraction of the diversity and relative 359 abundance (Table 1). Bacteria photoautotrophs were relatively more abundant than 
their protistan counterparts but less diverse (Table 1). Protistan parasites represented

$361 \sim 8 \%$ of the OTUs and $\sim 3 \%$ of the abundance, while the remaining protistan lifestyles

362 had a minor relevance in the core microbiota (Table 1).

Intra- and cross-domain core associations

365 Bacteria tended to be associated with other bacteria (Table $3 \&$ 4; Figure 2B), with

366 Bacteria-Bacteria associations making up $\sim 54 \%$ of all associations, while Protist-Protist

367 associations accounted for $11 \%$ (Table 4). The connectivity of the bacterial 368 subnetworks was higher (mean degree $\sim 10$ ) than the protist counterparts (mean degree $369 \sim 6$ ), regardless of whether these networks included exclusively bacteria, protists, or both (Table 3).

In particular, there was a substantial number of associations between Alpha-

372 and Gammaproteobacteria, between Alphaproteobacteria and Acidiimicrobia as well as

373 among Alphaproteobacteria OTUs (Figure 2B). Eukaryotic OTUs did not show a

374 similar trend with associations between OTUs of the same taxonomic ranks (Figure 375 2B). In terms of cross-domain associations, Alphaproteobacteria OTUs had several associations with most major protistan groups (i.e. dinoflagellates, diatoms,

377 cryptophytes, Mamiellophyceae, and Syndiniales) [Figure 2B].

Core associations within the pico- and within the nanoplankton

380 While the pico- and nano-size fractions indicate different lifestyles in bacteria (free-

381 living or particle-attached), they indicate different cell sizes in protists, and this could

382 be reflected in association networks. Nanoplankton sub-networks were larger and more 383 connected than picoplankton counterparts (Figure 4, Table 3). This pattern was 384 observed in both sub-networks considering associations from the same or both size 
385 fractions (Table 3). Nanoplankton sub-networks had a higher average degree $(\sim 10)$

386 than picoplankton sub-networks $(\sim 7$; Wilcoxon $\mathrm{p}<0.05)$, while not differing much in

387 other network statistics (Table 3). Most associations in the pico- and nanoplankton

388 were positive $(>93 \%)$, while the associations between OTUs from different size

389 fractions represented only $\sim 17 \%$ of the total, being $\sim 99 \%$ positive (Table 2 ).

In the pico- or nanoplankton sub-networks that include OTUs from the same

391 size fraction, the number of bacterial core OTUs was higher than the protistan

vs. 30 protistan OTUs in the picoplankton) (Figure 4, Table 3). Still, core OTUs in

both the pico- and nanoplankton had comparable sequence abundances: $\sim 27 \%$ of the

(Table 4). Overall, the BBMO pico- and nanoplankton sub-networks differed in size, connections and relative sequence abundance.

Table 3. Core network and sub-networks statistics.

\begin{tabular}{|c|c|c|c|c|c|c|c|c|c|}
\hline Network & $\begin{array}{c}\text { Nodes } \\
\text { (\#OTUs) }\end{array}$ & Edges & Di. & De. & $\begin{array}{l}\text { Average } \\
\text { degree }\end{array}$ & $\begin{array}{c}\text { Average } \\
\text { path } \\
\text { length }\end{array}$ & $\begin{array}{l}\text { Average } \\
\text { clustering } \\
\text { coefficient }\end{array}$ & $\begin{array}{c}\text { Largest } \\
\text { clique } \\
\text { (\#) }\end{array}$ & Mod. \\
\hline Core network & $262(259)$ & 1,411 & 11 & 0.04 & 10.7 & 3.45 & 0.52 & $13(4)$ & 0.19 \\
\hline Random core network & 262 & 1,411 & 5 & 0.04 & 10.7 & 2.60 & 0.03 & $3(199)$ & 0.13 \\
\hline Picoplankton all 1 & $161(160)^{*}$ & $620^{*}$ & 10 & 0.05 & 7.7 & 3.13 & 0.55 & $10(1)$ & 0.22 \\
\hline Picoplankton only ${ }^{2}$ & $110(109)$ & 378 & 9 & 0.06 & 6.9 & 3.15 & 0.51 & $9(4)$ & 0.29 \\
\hline Nanoplankton all 3 & $197(194)^{*}$ & $1,033^{*}$ & 10 & 0.05 & 10.5 & 3.18 & 0.57 & $13(4)$ & 0.15 \\
\hline Nanoplankton only 4 & $153(150)$ & 791 & 10 & 0.07 & 10.3 & 3.21 & 0.56 & $13(4)$ & 0.17 \\
\hline Bacteria all 5 & $233(230)^{\star *}$ & $1,236^{* *}$ & 10 & 0.04 & 10.6 & 3.34 & 0.52 & $11(3)$ & 0.19 \\
\hline Bacteria only 6 & $185(182)$ & 803 & 10 & 0.05 & 8.7 & 3.50 & 0.51 & $10(1)$ & 0.31 \\
\hline Protists all 7 & $147(145)^{\star *}$ & $608^{* *}$ & 5 & 0.06 & 8.3 & 2.40 & 0.48 & $8(2)$ & 0.10 \\
\hline Protist only ${ }^{8}$ & $80(77)$ & 175 & 5 & 0.05 & 4.4 & 2.54 & 0.54 & $7(1)$ & 0.32 \\
\hline
\end{tabular}

NB: Networks and sub-networks include OTUs and environmental factors. Di=Network diameter. De=Network density. Largest clique $=$ size of the largest clique(s) in the network, and in brackets, the number of them. Mod = Network modularity inferred using edge betweenness. ${ }^{1}$ All associations where picoplankton OTUs are involved (including nanoplankton); ${ }^{2}$ Associations between picoplankton OTU only; ${ }^{3} \mathrm{All}$ associations where nanoplankton OTUs are involved (including picoplankton); ${ }^{4}$ Associations between nanoplankton OTU only; ${ }^{5} \mathrm{All}$ associations where bacterial OTUs are involved (including protists); ${ }^{6}$ Associations between bacterial OTU only; ${ }^{7}$ All associations where protist OTUs are involved 
bioRxiv preprint doi: https://doi.org/10.1101/2021.03.18.435965; this version posted June 24, 2021. The copyright holder for this preprint (which was not certified by peer review) is the author/funder, who has granted bioRxiv a license to display the preprint in perpetuity. It is made available under aCC-BY-NC-ND 4.0 International license.

410

411

412

(including bacteria); ${ }^{8}$ Associations between protist OTU only. * Includes nodes and edges shared between pico- and nanoplankton. ${ }^{*}$ Includes nodes and edges shared between bacteria and protists.

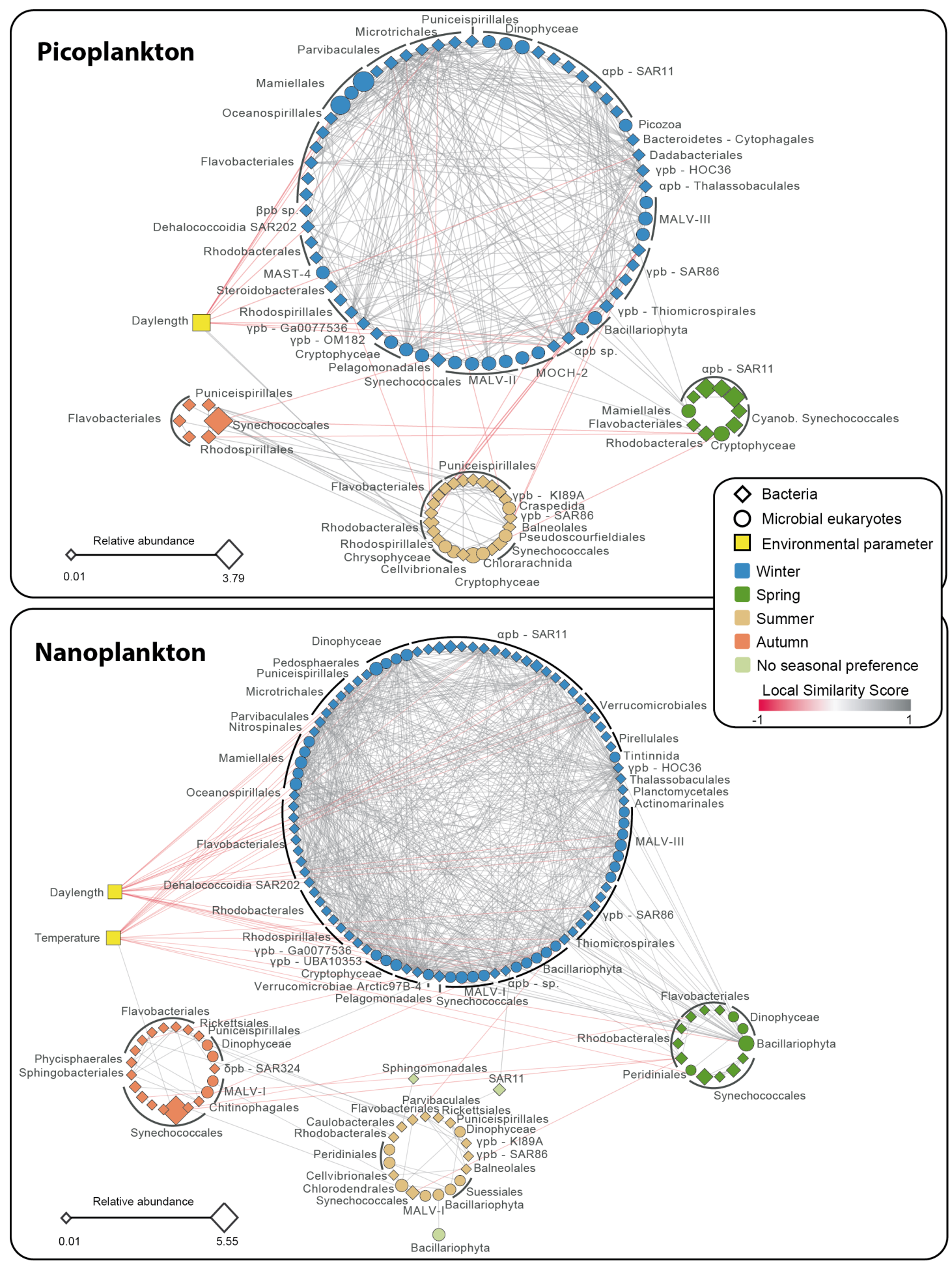

415 Figure 4. Pico- and nanoplankton core sub-networks. The shape of the nodes indicates

416 bacteria (rhomboid) or microbial eukaryotes (circle), and the color of nodes represents species 
Table 4. Core associations within and between taxonomic domains and size fractions.

\begin{tabular}{llc}
\hline \multicolumn{1}{c}{ Network } & Association type $^{1}$ & \# Associations \\
\hline Core network & Total & 1,411 \\
& Bacteria - Bacteria & $767(54 \%)$ \\
& Bacteria - Protist & $433(31 \%)$ \\
& Protist - Protist & $161(11 \%)$ \\
& Environmental factor - Bacteria & $36(3 \%)$ \\
& Environmental factor - Protist & $14(1 \%)$ \\
Picoplankton subnetwork & Total & 378 \\
& Bacteria - Bacteria & $241(64 \%)$ \\
& Bacteria - Protist & $94(25 \%)$ \\
& Protist - Protist & $31(8 \%)$ \\
& Environmental factor - Bacteria & $12(3 \%$ \\
& Environmental factor - Protist & $0(0 \%)$ \\
Nanoplankton subnetwork & Total & 791 \\
& Bacteria - Bacteria & $394(50 \%)$ \\
& Bacteria - Protist & $246(31 \%)$ \\
& Protist - Protist & $113(14 \%)$ \\
& Environmental factor - Bacteria & $24(3 \%)$ \\
& Environmental factor - Protist & $14(2 \%)$ \\
\hline
\end{tabular}

1"Bacteria - Bacteria" indicates associations between two bacterial OTUs. "Protist - Protist" are associations between two unicellular eukaryotes and "Bacteria - Protist" are associations between one eukaryote and one bacterial OTU. "Environmental factor - Protist" and "Environmental factor - Bacteria" are associations between an environmental factor and a eukaryotic or bacterial OTU.

Network seasonality

427 The indicator value (IndVal) was used to infer the seasonal preference of core OTUs. Most of the core OTUs (98\%; 254 out of 259 OTUs) showed a clear preference for one

429 of the four seasons, pointing to a marked seasonality in the core microbiota (Figure 4;

430 Table 5; Tables S4 \& S5, Additional file 1). Winter had the highest quantity of core

431 OTUs and the highest network connectivity (average degree $\sim 13$ ), compared to the 432 other seasons (average degrees $\sim 2-\sim 6$ ) [Figure 4; Table 5]. The average path length 433 was larger in the core network compared to a random network of the same size (Table 434 3). Yet, all sub-networks associated with size fractions and seasons (Table 5) had 435 shorter path lengths than the random network, indicating that nodes tended to be 
connected within seasons and size fractions. This was also supported by an increase in

437 network density when comparing the core network (Table 3) and the core network

438 subdivided into seasons (Table 5), against the core network subdivided into both

439 seasons and size fractions (Table 5). The five OTUs that did not show any seasonal

440 preference, among them SAR11 Clades Ia \& II, showed high to moderate abundances

441 but had a low number of associations to other OTUs (Tables S4, S5, S6, Additional

442 file 1). Thus, network connectivity in the BBMO appears to be heterogeneous over

443 time, peaking in winter and remaining low in the other seasons.

Table 5: Subnetworks including core OTUs displaying seasonal preference.

\begin{tabular}{|c|c|c|c|c|c|c|c|c|c|c|}
\hline & $\begin{array}{c}\text { Sub- } \\
\text { network }\end{array}$ & $\begin{array}{c}\text { Number of } \\
\text { OTUs }\end{array}$ & Edges & Di. & De. & $\begin{array}{l}\text { Average } \\
\text { degree }\end{array}$ & $\begin{array}{l}\text { Average } \\
\text { path } \\
\text { length }\end{array}$ & $\begin{array}{l}\text { Average } \\
\text { clustering } \\
\text { coefficient }\end{array}$ & $\begin{array}{l}\text { Largest } \\
\text { clique } \\
\text { (\#) }\end{array}$ & Mod. \\
\hline \multirow{4}{*}{$\bar{\varepsilon}$} & Winter & 156 & 1,175 & 7 & 0.10 & 15.1 & 2.62 & 0.54 & $13(4)$ & 0.19 \\
\hline & Spring & 19 & 16 & 4 & 0.09 & 1.7 & 1.56 & 0.44 & $4(1)$ & 0.75 \\
\hline & Summer & 41 & 56 & 7 & 0.07 & 2.7 & 2.90 & 0.49 & $6(1)$ & 0.53 \\
\hline & Autumn & 26 & 25 & 3 & 0.08 & 1.9 & 1.59 & 0.46 & $4(2)$ & 0.73 \\
\hline \multirow{4}{*}{$\frac{0}{2}$} & Winter & 63 & 286 & 6 & 0.15 & 9.1 & 2.35 & 0.53 & $9(4)$ & 0.10 \\
\hline & Spring & 8 & 5 & 3 & 0.18 & 1.2 & 1.50 & 0.00 & $2(5)$ & 0.56 \\
\hline & Summer & 25 & 36 & 5 & 0.12 & 2.9 & 2.20 & 0.41 & $6(1)$ & 0.23 \\
\hline & Autumn & 5 & 3 & 2 & 0.30 & 1.2 & 1.25 & 0.00 & $2(3)$ & 0.44 \\
\hline \multirow{4}{*}{ 茮 } & Winter & 92 & 658 & 6 & 0.16 & 14.3 & 2.40 & 0.61 & $13(4)$ & 0.04 \\
\hline & Spring & 11 & 11 & 4 & 0.20 & 2.0 & 1.59 & 0.57 & $4(1)$ & 0.56 \\
\hline & Summer & 13 & 17 & 3 & 0.22 & 2.6 & 1.70 & 0.65 & $4(1)$ & 0.50 \\
\hline & Autumn & 17 & 18 & 3 & 0.13 & 2.1 & 1.35 & 0.56 & $4(2)$ & 0.60 \\
\hline
\end{tabular}

NB: Subnetworks include OTUs only. Di=Network diameter. De=Network density. Largest clique = size of the largest clique(s) in the network,

451 Within the core network, we identified groups that were more connected to each other

452 than to the rest of the network (called modules). These groups of OTUs may indicate

453 recurring associations that are likely important for the stability of ecosystem function.

454 We identified 12 modules in both the pico- and nanoplankton subnetworks (Table S7,

455 Additional file 1). Modules tended to include OTUs from the same season (Table S8,

456 Additional file 1), with main modules (i.e. MCODE score $>4$ ) including OTUs

457 predominantly associated with winter, summer, and autumn (Figure 5). Overall, winter 
modules prevailed (5 out of 7) among the main modules (Figure 5), while modules

with scores $\leq 4$ did not tend to be associated with a specific season (Table S8,

Additional file 1). Two main winter modules had members that were negatively correlated to temperature and daylength (Figure 5; Modules 1 and 4, nanoplankton). $\sim 24 \%$ (proportional to the resident microbiota), while the total abundance of individual modules ranged between $\sim 6 \%$ and $\sim 0.3 \%$ (Table S7, Additional file 1). In turn, the relative abundance of core OTUs included in modules ranged between $0.01 \%$ and $\sim 2 \%$

466 (Table S8, Additional file 1). In most modules, a few OTUs tended to dominate the 467 abundance, although there were exceptions, such as module 4 of the picoplankton, where all SAR11 members featured abundances $>1 \%$ (Table S8, Additional file 1). In addition, several OTUs within modules had relatively low abundances (Table S8, Additional file 1), supporting modules as a real feature of the network and not just the

471 agglomeration of abundant taxa.

\section{Central OTUs}

474 Biological networks typically contain nodes (i.e. OTUs) that hold more "central" 475 positions in the network than others [22]. Even though the ecological role of these hub 476 and connector OTUs is unclear, it is acknowledged that they could reflect taxa with 477 important ecological functions [22]. There is no universal definition for hub or 478 connector OTUs, yet, in this work, we have used stringent thresholds to determine them 479 ad hoc (see Methods). We have identified 13 hub-OTUs that were associated with 480 winter or spring (Table 6). Hubs did not include highly abundant OTUs, such as 481 Synechococcus or SAR11 (Table 6), but instead, they included several OTUs with 482 moderate-low abundance $(<1 \%)$ and high degree (ranging between 26-60) [Table 6]. 
483 For example, the Gammaproteobacteria OTU bn_000226 had a relative abundance of

$484 \quad 0.04 \%$ and a degree of 60 (Table 6). Hubs included other moderately abundant OTUs,

485 such as the eukaryotic picoalgae Bathycoccus, which was abundant in winter, as well 486 as an unidentified dinoflagellate (Table 6).

487 We identified a total of 18 connector OTUs (featuring relatively low degree and 488 high centrality), which were predominantly associated with summer (5 out of 18) or 489 autumn (6 out of 18), contrasting with hub OTUs, which were associated mostly with 490 winter and spring (Table 6). Connectors may be linked to the seasonal transition 491 between main community states (Figure 1 C \& D) and included several abundant 492 OTUs belonging to Synechococcus and SAR11 (Table 6). In particular, the SAR11 493 OTU bp_000007 displayed a relatively high abundance (1.4\%), but a degree of 3 494 (relatively low) and a betweenness centrality of 0.6 (relatively high). In contrast, two 495 protist OTUs displayed low-moderate abundances (ep_00269, Chrysophyceae, 496 abundance $0.04 \%$ and en_00161, Syndiniales, abundance $0.4 \%$ ), low degree $<4$, but a 497 high betweenness centrality $(>0.8$; Table 6$)$. 
bioRxiv preprint doi: https://doi.org/10.1101/2021.03.18.435965; this version posted June 24, 2021. The copyright holder for this preprint

(which was not certified by peer review) is the author/funder, who has granted bioRxiv a license to display the preprint in perpetuity. It is made available under aCC-BY-NC-ND 4.0 International license.

508

509 Table 6. Central OTUs.

\begin{tabular}{|c|c|c|c|c|c|c|c|}
\hline OTU & Class & Lowest rank taxonomy & $\begin{array}{c}\text { Relative } \\
\text { Abundance (\%) } \\
\end{array}$ & Degree & $\begin{array}{l}\text { Betweenness } \\
\text { Centrality }\end{array}$ & $\begin{array}{l}\text { Closeness } \\
\text { Centrality }\end{array}$ & Season \\
\hline \multicolumn{8}{|c|}{ Hubs } \\
\hline en_00092 & Mamiellophyceae & Bathycoccus & 0.51 & 42 & 0.04 & 0.42 & Winter \\
\hline en_00119 & Dinophyceae & - & 0.41 & 50 & 0.03 & 0.42 & Winter \\
\hline bp_000037 & Alphaproteobacteria & Parvibaculales_OCS116 & 0.31 & 45 & 0.08 & 0.43 & Winter \\
\hline bp_000039 & Gammaproteobacteria & SUP05_cluster & 0.28 & 29 & 0.12 & 0.41 & Spring \\
\hline bn_000039 & Gammaproteobacteria & SUP05_cluster & 0.21 & 42 & 0.17 & 0.44 & Spring \\
\hline bn_000037 & Alphaproteobacteria & Parvibaculales_OCS116 & 0.20 & 40 & 0.05 & 0.42 & Spring \\
\hline bp_000059 & Gammaproteobacteria & SAR86 & 0.20 & 24 & 0.09 & 0.40 & Spring \\
\hline ep_00070 & Cryptophyceae & Cryptomonadales_X & 0.13 & 40 & 0.04 & 0.42 & Winter \\
\hline bn_000059 & Gammaproteobacteria & SAR86 & 0.12 & 24 & 0.03 & 0.40 & Spring \\
\hline bn_000102 & Alphaproteobacteria & Nisaeaceae_OM75 & 0.09 & 26 & 0.03 & 0.38 & Winter \\
\hline bp_000193 & Alphaproteobacteria & - & 0.06 & 37 & 0.03 & 0.40 & Winter \\
\hline bn_000170 & Acidimicrobiia & Sva0996_marine_group & 0.06 & 59 & 0.06 & 0.44 & Winter \\
\hline bn_000226 & Gammaproteobacteria & HOC36 & 0.04 & 60 & 0.06 & 0.43 & Winter \\
\hline \multicolumn{8}{|l|}{ Connectors } \\
\hline bp_000001 & Oxyphotobacteria & Synechococcus (CC9902) & 3.79 & 5 & 0.05 & 0.30 & Autumn \\
\hline bp_000002 & Alphaproteobacteria & SAR11 Clade_la & 2.26 & 2 & 0.40 & 0.56 & Spring \\
\hline bp_000004 & Alphaproteobacteria & SAR11 Clade_la & 2.02 & 3 & 0.15 & 0.63 & NA \\
\hline bp_000007 & Alphaproteobacteria & SAR11 Clade_la & 1.38 & 3 & 0.60 & 0.71 & NA \\
\hline bp_000008 & Alphaproteobacteria & SAR11 Clade_la & 1.15 & 3 & 0.15 & 0.63 & NA \\
\hline bn_000008 & Alphaproteobacteria & SAR11 Clade_la & 0.68 & 5 & 0.03 & 0.27 & Winter \\
\hline en_o0059 & Chlorodendrophyceae & Tetraselmis & 0.66 & 4 & 0.05 & 0.26 & Summer \\
\hline bn_000020 & Oxyphotobacteria & - & 0.56 & 3 & 0.60 & 0.67 & Autumn \\
\hline en_o0161 & Syndiniales & Syndiniales-Group-I-Clade-4_X & 0.42 & 4 & 0.80 & 0.75 & Autumn \\
\hline bn_000018 & Oxyphotobacteria & Prochlorococcus MIT9313 & 0.41 & 5 & 0.04 & 0.24 & Winter \\
\hline bn_000054 & Alphaproteobacteria & Puniceispirillales_SAR116 & 0.11 & 4 & 0.14 & 0.40 & Autumn \\
\hline bn_000062 & Alphaproteobacteria & Puniceispirillales_SAR116 & 0.08 & 3 & 0.55 & 0.50 & Autumn \\
\hline bn_000077 & Rhodothermia & Balneola & 0.07 & 3 & 0.17 & 0.32 & Summer \\
\hline bn_000112 & Gammaproteobacteria & $\mathrm{Kl} 189 \mathrm{~A}$ & 0.06 & 4 & 0.53 & 0.48 & Summer \\
\hline bn_000156 & Alphaproteobacteria & Parvibaculales_PS1 & 0.05 & 4 & 0.14 & 0.40 & Summer \\
\hline bn_000281 & Bacteroidia & Sphingobacteriales_NS11-12 & 0.05 & 5 & 0.16 & 0.44 & Autumn \\
\hline bn_ 000221 & Alphaproteobacteria & Puniceispirillales_SAR116 & 0.04 & 5 & 0.05 & 0.30 & Winter \\
\hline ep_o0269 & Chrysophyceae & Clade-I_X & 0.04 & 2 & 1.00 & 1.00 & Summer \\
\hline
\end{tabular}

\section{1}


bioRxiv preprint doi: https://doi.org/10.1101/2021.03.18.435965; this version posted June 24, 2021. The copyright holder for this preprint (which was not certified by peer review) is the author/funder, who has granted bioRxiv a license to display the preprint in perpetuity. It is made available under aCC-BY-NC-ND 4.0 International license.

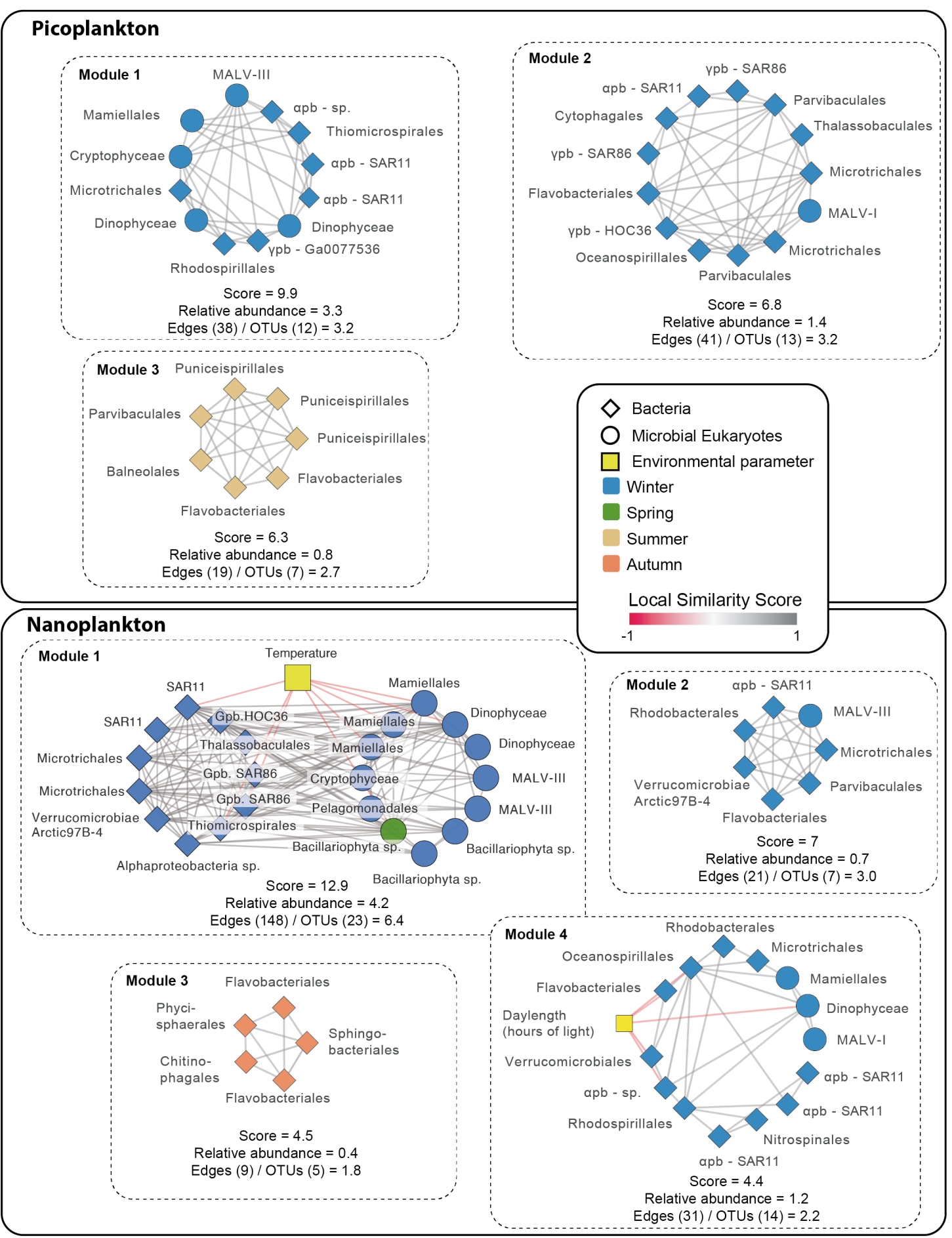

Figure 5. Main modules in the core network. Modules with MCODE score $>4$ are shown for

515 picoplankton (upper panel) and nanoplankton (lower panel). For each module, the MCODE score

516 and relative amplicon abundance of the taxa included in it (as \% of the resident microbiota) are

517 indicated. In addition, the numbers of edges and OTUs within the modules are shown as

518 edges/OTUs; this quotient estimates the average number of edges per OTU within the different 
519 modules. The edges represent correlations with $|\mathrm{LS}|>0.7,|\rho|>0.7, \mathrm{P}<0.001$ and $\mathrm{Q}<0.001$. The

520 color of the edges indicates positive (grey) or negative (red) associations. The shape of nodes

521 indicates bacteria (rhomboid) or microbial eukaryotes (circle), and the color of nodes represents

522 species seasonal preferences, determined using the indicator value $(p<0.05)$. $p b=$

523 Proteobacteria

\section{DISCUSSION}

527 Identifying the most important microbes for the functioning of the ocean ecosystem is

528 a challenge, which can be addressed by delineating core microbiotas [4]. Recognizing

529 the most abundant and widespread microbes in the ocean is a step towards knowing the

530 core microbiota. However, this does not take into account the importance that both

531 microbial interactions and microbes with moderate or low abundance may have for the

532 functioning of ecosystems [4, 29, 38]. Considering potential interactions when

533 delineating core microbiotas may not only allow identifying moderate/low abundance

534 taxa that may have important roles in the community but could also allow excluding

535 taxa that are present in several locations but that may not have an important role for

536 community function (e.g., dormant cells or cells being dispersed [8]). Here, we have

537 delineated and analyzed the core microbiota of a coastal ecosystem-based on 10 years

538 of occurrence data considering possible interactions.

539 To detect the core microbiota, we first identified the resident OTUs, that is,

540 those that occur $>30 \%$ of the time (i.e. $>36$ out of 120 months) over a decade. This

541 threshold was selected as it allows for seasonal OTUs that would be present recurrently

542 in at least one season. Analysis of the resident OTU dynamics indicated a clear

543 seasonality (Figure 1 C-D), and that the measured environmental factors could explain

$544 \sim 45 \%$ of the resident microbiota variance. The main environmental drivers were 
temperature and daylength, which is consistent with previous works from the same time-series (BBMO) $[34,39,40]$. These values are lower than what has been reported

547 for bacteria in the English Channel, where daylength explains $\sim 65 \%$ of community

548 variance [17], and higher than what has been reported for entire communities in the

549 time-series SPOT (California, 31\%) [41] or SOLA (the Mediterranean Sea, $\sim 130 \mathrm{~km}$

550 from BBMO; 7-12\%) [42]. Daylength may be more important in the English Channel

551 as it has a more pronounced annual variation than at BBMO, whereas the measured

552 differences could reflect a higher coupling of the resident OTUs with environmental

553 variation in BBMO than in SOLA or SPOT. SOLA is characterized by the occasional

554 winter storms that bring nutrients from the sediments to the water column as well as by

555 the freshwater inputs from nearby rivers during flash floods [43], and this could

556 partially explain the differences with BBMO. The importance of daylength and

557 temperature for community dynamics was reflected by niche analyses, which identified

558 two main niches associated with summer and winter at the BBMO, to which $\sim 50 \%$ of

559 the resident OTUs were associated (Figure 1 E-F). Other resident OTUs likely have

560 spring and fall niches as indicated by Figure 1 C-D, yet these niches cannot be detected

561 with the used null model analysis, as their preferred temperatures or daylengths will not

562 depart significantly from the randomized mean.

563 Based on the resident OTUs, we built networks to define the core microbiota.

564 We identified a total of 259 core OTUs (182 bacteria and 77 protists) that represented

$56564 \%$ of the abundance of the resident microbiota and that showed seasonal variation.

566 We could only find supporting evidence from the literature (PIDA database) [21] for

56785 associations of the core $(6 \%)$, indicating that most of them still need to be validated

568 with direct observation or experimentally. This is not surprising, as the most studied

569 hosts in PIDA are protists from the micro-plankton ( $>20 \mu \mathrm{m}$ cell size), which are mostly 
absent from our pico- and nanoplankton networks. Also, PIDA does not cover Bacteria-

571 Bacteria associations. Nevertheless, the detected core OTUs from BBMO represent a

572 fraction of the core microbiota at this site, since larger microbial size fractions were not

573 sampled. Including these larger size fractions would expand the composition of the core

574 and could unveil additional patterns. For example, in a global ocean network including

575 size fractions $>20 \mu \mathrm{m}$ cell size, protists or small multicellular eukaryotes dominated the 576 interactome [26].

Alpha-/Gammaproteobacteria, Bateroidia, Acidimicrobiia were the main

578 bacterial groups in the core, including also common marine taxa, such as

579 Synechococcus or SAR11. The main protists in the core included Syndiniales

580 (parasites), Dinoflagellates, Mammiellales (Micromonas and Bathycoccus), and

581 diatoms. These taxa are likely the most important in sustaining ecosystem function at

582 BBMO, and probably have similar importance in other coastal areas. Other studies have

583 reported important roles in marine association networks for SAR11 and Synechococcus

$584[31,44]$. Syndiniales, Haptophytes, and Dinoflagellates dominated networks in terms

585 of the number of nodes and edges at SPOT, while Mamiellales (Micromonas \&

586 Bathycoccus) and diatoms also had relevant roles [41]. Syndiniales, Dinoflagellates,

587 and Diatoms were also predominant in global ocean networks, which is coherent with

588 our results [26].

589 Bacteria-Bacteria associations were the most abundant (54\%) in the core

590 BBMO microbiota, followed by Bacteria-Protists (31\%) and Protist-Protist (11\%)

591 associations. Associations tended to occur among bacteria or protists, rather than

592 between them, in the English Channel time-series [17]. However, the study used

593 microscopy to determine protist community composition, while it used 16S-rRNA gene

594 data for analyzing bacteria communities and this might explain the limited number of 
connections between protists and bacteria. Most associations occurred among protists higher proportion of associations among protists, which may turn out to be prevalent. coefficient and relatively short path lengths) [37] when compared to randomized networks (Table 3) or particular subnetworks from size fractions or specific seasons

604 31]. Some of our network statistics were similar to those obtained at SPOT [23, 30], in 605 particular the averages of degree, clustering coefficient, and path length (Table 3). 606 Furthermore, the BBMO network had an average path length similar to a global ocean 607 network [26] and also, similarly to this network, the node degree of the BBMO core 608 members was independent of their relative abundances, showing that the associations 609 between core OTUs were not merely a consequence of high prevalence and abundance.

614 or large-scale spatial [26] microbiota analyses, where positive associations were also 615 predominant $(\sim 70-98 \%)$, although these values include taxa that are not necessarily part 616 of the core. This suggests that interactions such as syntrophy or symbiotic associations 617 are more important than competition in marine microbial systems and that these types 618 of associations may underpin marine ecosystem function. These findings are also 619 coherent with a recent large-scale literature survey that found that $\sim 47 \%$ of the validated 
associations between protists and bacteria are symbiotic [21]. Nevertheless, it is also

621 possible that common sampling strategies and methodological approaches do not detect

622 a substantial fraction of negative associations. For example, while positive correlations

623 in taxa abundance pointing to positive interactions may be easier to detect, negative

624 associations may be missed due to plummeting species abundances that would prevent

625 establishing significant correlations, or to a delay between the increase and decrease in

626 abundance of interacting taxa that are not synchronized with sampling time. Future

627 studies adapting the sampling scheme to the timing of interactions (e.g., daily or weekly

628 sampling) and the use of other approaches apart from taxa abundances, such as analyses

629 of single-cell genomic data to determine protistan predation, or controlled experiments,

630 will likely generate new insights on negative microbial interactions.

The relatively high clustering coefficient of the core network (compared to a

632 random network) and its short path length indicate that most OTUs are connected

633 through $<3$ intermediary OTUs. It has been shown that a large proportion of strong

634 positive associations, as in the BBMO core network, may destabilize communities due

635 to positive feedbacks between species [46]. When a species decreases in abundance as

636 a response to environmental variation, it may pull others with it, generating a cascade

637 effect propagated by the many positive associations in the network. Accordingly, the

638 change of abundance in specific OTUs in one section of the network could affect OTUs

639 in other network sections not necessarily affected directly by the environmental

640 variation. This cascade effect may help to explain a paradox: environmental variables

641 affect the structure of marine microbial communities and consequently association

642 networks. Yet, our and others' results [17, 18, 23, 26, 30-32] have reported a limited

643 number of associations between environmental variables and network nodes (OTUs).

644 Environmental heterogeneity might affect network structure by acting on a small subset 
645 of nodes (OTUs), which would then influence other nodes through cascading

646 interactions facilitated by the highly interconnected nature of the networks as well as

647 positive feedbacks promoted by the high proportion of positive associations [46].

648 If OTUs susceptible to environmental variation are also highly connected, then

649 their effect on the entire network structure may be larger. In line with this, we found 650 that the connectivity of OTUs associated with environmental variables at BBMO (49

651 OTUs out of 259) had a mean degree of $\sim 25$ (SD $\sim 14$ ), while for all the 259 OTUs of

652 the core network, the mean degree was $\sim 11$ (SD $\sim 13)$. The seasonal dynamics of the 653 BBMO microbiota may partially be driven by a subset of OTUs that vary with 654 environmental factors (e.g. temperature, daylength). These may exert a destabilizing 655 influence over the entire community over time, promoting the annual turnover of communities and networks.

Most core OTUs (98\%) showed a clear preference for one season. Interestingly,

658 the distribution of core OTUs among the seasons was uneven, with $61 \%$ of these OTUs

659 showing a winter preference. Network connectivity at BBMO was correspondingly

660 heterogeneous between seasons, peaking in winter and remaining low in the other 661 seasons. Specifically, the winter subnetwork included $\sim 92 \%$ of the seasonal edges. This

662 indicates that winter associations are not only specific (i.e. they do not tend to change 663 partners), but they also have a relatively high recurrence (otherwise, winter networks 664 would be smaller). A higher similarity between winter communities when compared to 665 other seasons was also indicated by our ordination analyses of the resident OTUs 666 (Figure 1), as well as by studies of the entire protist community at BBMO [34] or whole 667 community analyses at SPOT [23].

668 The structure of communities is determined by the interplay of selection, 669 dispersal, speciation, and ecological drift [47]. Our results indicate that selection, a 
deterministic process, is stronger in winter, leading to winter sub-communities that tend

671 to be more similar between each other than to communities from other seasons. Given

672 that we have removed edges associated with the measured environmental variables, we

673 do not expect that the identified edges between winter OTUs represent selection

674 associated to these variables (e.g. low temperature). Consequently, winter edges may represent associations linked to unmeasured variables or ecological interactions that may be more likely to develop during winter due to stronger environmental selection.

677 Due to weaker selection in other seasons species occurrence would display less 678 recurrent (or more random) patterns, preventing specific associations to be formed. This 679 also suggests that ecological redundancy changes over time, and is lower in winter compared to the other seasons (even though the number of OTUs is larger in winter).

681 A reduction in redundancy may also promote strong ecological interactions in winter. than with other species (modules), is characteristic of biological networks, and can 684 contribute to overall network stability [48, 49]. Modules can represent divergent 685 selection, niches, the clustering of evolutionary closely related species or coevolutionary units $[50,51]$. Modules in the core BBMO network (total 12) included positive associations between diverse taxa, and could represent divergent selection,

688 driven by unmeasured environmental variables, or examples of syntrophic or symbiotic 689 interactions between microbes from different taxonomic groups.

Most BBMO modules included diverse lifestyles (heterotrophs, mixotrophs, 691 phototrophs, parasites), similar to what has been observed at SPOT [41]. Yet, a number 692 of modules appeared to be predominantly heterotrophic or autotrophic (Table S8, 693 Additional file 1). Some modules included OTUs from the same species, such as 694 Module 4 in the picoplankton, which included several SAR11 Clade I OTUs, and 
695 Module 7 of the nanoplankton, which included several Synechococcus OTUs. These modules could reflect similar niches, associated with unmeasured variables, or the

697 dependence on metabolites produced by other organisms (auxotrophy). There is 698 evidence of auxotrophy for both SAR11 (e.g. thiamin, glycine)[52-54] and 699 Synechococcus (e.g. cobalamin) [55]. Recently it has been observed in co-culture experiments that Prochlorococcus may fulfill some metabolic requirements of SAR11, 701 promoting the growth of the latter in a commensal relationship [56]. In our analyses of 702 the BBMO core microbiota, we did not find strong associations between SAR11 and 703 Prochlorococcus or the more abundant relative, Synechococcus. Yet, SAR11 formed 704 strong associations with a plethora of taxa with which could potentially have 705 commensal relationships. The overall importance of the observed modules was indicated by the total 707 abundance of their constituent OTUs $(24 \%$ of the reads compared to the resident 708 microbiota). Most of the modules at BBMO were associated with a single season, 709 suggesting that they reflect seasonal niches. Since these modules were inferred over 10 710 years, they represent recurrent network features. Chafee et al. [57] also identified 711 season-specific modules in a 2-year time series in the North Sea (Helgoland), including 712 samples taken weekly or bi-weekly. These modules were much larger than ours, and 713 they may also include environmentally-driven edges. Nevertheless, the Helgoland 714 modules seem to be driven by eutrophic (spring \& summer) vs. oligotrophic (autumn $715 \&$ winter) conditions in this location. In contrast, the BBMO modules, displayed weaker 716 correlations with nutrients and seem to be influenced by temperature and daylength

717 (Figure 5). Differences in the sampling scheme between Helgoland and BBMO 718 ((bi)weekly vs. monthly) as well as between both locations (different seas and latitudes, 719 affecting temperature and daylength) may explain these differences. 
Keystone species have a high influence in ecosystems relative to their

721 abundance [58]. Network analyses may help to identify them [24, 59], yet, there is no

722 clear consensus of what network features are the best unequivocal indicator of keystone

723 species [60-62]. Therefore, we focused on identifying central OTUs (hubs or

724 connectors) that may be important for ecosystem function $[22,24]$ and could represent

725 keystone species. We identified 13 hubs in the BBMO core network with moderate-low

726 abundances $(<1 \%)$ and high degree (26-60) that were associated with winter or spring.

727 These moderate-low abundance OTUs may affect nutrient cycling directly [63] or

728 indirectly, by affecting other OTUs with higher abundance. The putative stronger

729 selection exerted by low temperatures and short daylengths during winter and early

730 spring, as compared to summer and autumn, may lead to a higher species recurrence

731 [34], larger networks, and possibly, more hubs. An OTU of the abundant picoalgae

732 Bathycoccus (en_00092) was identified as a winter hub, which is consistent with

733 reported Bathycoccus abundance peaks in late winter (February-March) in both BBMO

734 [64] and the nearby station SOLA [42]. This Bathycoccus hub may be associated with

735 diverse taxa, such as prokaryotes that may benefit from algal exudates [65] or even via

736 mixotrophy [66]. In agreement with this, out of the 42 associations of this hub OTU,

73725 were with bacteria and the rest with protists.

738 In contrast to hubs, connector OTUs were predominantly associated with

739 warmer waters, that is, summer and autumn, and may represent transitions in

740 community states. This was consistent with the associations observed in an abundant

741 Synechococcus connector OTU (bp_000001, Table 6). This OTU was predominant in

742 summer-autumn, in agreement with previous BBMO reports [36, 67], but it was

743 associated with other OTUs from spring (negative association with bp_000017), winter

744 (negative association with bp_000039), summer (positive association with bp_000087, 
bp_000012) and autumn (positive association with bp_000022), thus likely holding a bp_000007) and summer (positive association with bp_000046) OTUs.

\section{CONCLUSION}

751 Our decade-long analysis of the dynamics of a microbiota populating a time-series in

752 the Mediterranean Sea allowed us to determine the interconnected core microbiota,

753 which likely includes several microbes that are important for the functioning of this

754 coastal ecosystem. We found a relatively small core microbiota that displayed seasonal

755 variation, with a heterogeneous distribution of associations over different seasons,

756 indicating different degrees of recurrence and selection strength over the year. Future

757 analyses of other core marine microbiotas will determine how universal are the patterns

758 found in BBMO. These studies will be crucial to determine potential long-term effects

759 of climate change on the architecture of the interaction networks that underpin the

760 functioning of the ocean ecosystem.

METHODS

763 Study site and sampling

764 Surface water $(\sim 1 \mathrm{~m}$ depth) was sampled monthly from January 2004 to December

7652013 at the Blanes Bay Microbial Observatory (BBMO) in the Northwestern

766 Mediterranean Sea $\left(41^{\circ} 40^{\prime} \mathrm{N}, 2^{\circ} 48^{\prime} \mathrm{E}\right)$ [Figure 1A]. The BBMO is an oligotrophic

767 coastal site $\sim 1 \mathrm{~km}$ offshore with $\sim 20 \mathrm{~m}$ depth and with limited riverine or human

768 influence [36]. Seawater was pre-filtered with a $200 \mu \mathrm{m}$ nylon mesh and then 769 transported to the laboratory in $20 \mathrm{~L}$ plastic carboys and processed within 2 hours. 
Microbial plankton from about $6 \mathrm{~L}$ of the pre-filtered seawater was separated into two

771 size fractions: picoplankton $(0.2-3 \mu \mathrm{m})$ and nanoplankton fraction $(3-20 \mu \mathrm{m})$. To

772 achieve this, the seawater was first filtered through a $20 \mu \mathrm{m}$ nylon mesh using a

773 peristaltic pump. Then the nanoplankton $(3-20 \mu \mathrm{m})$ was captured on a $3 \mu \mathrm{m}$ pore-size

774 polycarbonate filter. Subsequently, a $0.2 \mu \mathrm{m}$ pore-size Sterivex unit (Millipore,

775 Durapore) was used to capture the picoplankton $(0.2-3 \mu \mathrm{m})$. Sterivex units and $3 \mu \mathrm{m}$

776 filters were stored at $-80{ }^{\circ} \mathrm{C}$ until further processed. The sequential filtering process

777 aimed to capture free-living bacteria and picoeukaryotes in the $0.2-3 \mu \mathrm{m}$ size fraction

778 (picoplankton), and particle/protist-attached bacteria or nanoeukaryotes in the 3-20 $\mu \mathrm{m}$

779 fraction (nanoplankton). The $3 \mu \mathrm{m}$ filter was replaced if clogging was detected; DNA

780 from all $3 \mu \mathrm{m}$ filters from the same sample were extracted together.

A total of 15 contextual abiotic and biotic variables were considered for each

783 Secchi disk depth [m]), Salinity, Total Chlorophyll a [Chla] $(\mu \mathrm{g} / \mathrm{l}), \mathrm{PO}_{4}{ }^{3-}(\mu \mathrm{M}), \mathrm{NH}_{4}{ }^{+}$

$784(\mu \mathrm{M}), \mathrm{NO}_{2}^{-}(\mu \mathrm{M}), \mathrm{NO}_{3}^{-}(\mu \mathrm{M}), \mathrm{SiO}_{2}(\mu \mathrm{M})$, abundances of Heterotrophic prokaryotes

785 [HP] (cells/ml), Synechococcus (cells/ml), Total photosynthetic nanoflagellates [PNF;

$7862-5 \mu \mathrm{m}$ size] (cells $/ \mathrm{ml})$, small PNF $(2 \mu \mathrm{m}$; cells $/ \mathrm{ml})$ and, Heterotrophic nanoflagellates

$787[\mathrm{HNF}](\mathrm{cells} / \mathrm{ml})[$ Figure 1B]. Water temperature and salinity were sampled in situ with

788 a SAIV A/S SD204 CTD. Inorganic nutrients $\left(\mathrm{NO}_{3}{ }^{-}, \mathrm{NO}_{2}{ }^{-}, \mathrm{NH}_{4}{ }^{+}, \mathrm{PO}_{4}{ }^{3-}, \mathrm{SiO}_{2}\right)$ were

789 measured using an Alliance Evolution II autoanalyzer [68]. Cell counts were done by

790 flow cytometry (heterotrophic prokaryotes, Synechococcus) or epifluorescence

791 microscopy (PNF, small PNF and HNF). See Gasol et al. [36] for specific details on

792 how other variables were measured. Environmental variables were z-score standardized

793 before running statistical analysis. 
DNA extraction, sequencing, and metabarcoding

DNA was extracted from the filters using a standard phenol-chloroform protocol [69], purified in Amicon Units (Millipore), and quantified and qualitatively checked with a NanoDrop 1000 Spectrophotometer (Thermo Fisher Scientific). Eukaryotic PCR amplicons were generated for the V4 region of the 18S rDNA ( $\sim 380 \mathrm{bp})$, using the primer pair TAReukFWD1 and TAReukREV3 [70]. The primers Bakt_341F [71] and Bakt 806RB [72] were used to amplify the V4 region of the $16 \mathrm{~S}$ rDNA. PCR amplification and amplicon sequencing were carried out at the Research and Testing Laboratory (http://rtlgenomics.com/) on the Illumina MiSeq platform (2x250 bp pairedend sequencing). DNA sequences and metadata are publicly available at the European Nucleotide Archive (http://www.ebi.ac.uk/ena; accession numbers PRJEB23788 for 18S rRNA genes \& PRJEB38773 for 16S rRNA genes).

A total of 29,952,108 and 16,940,406 paired-end Illumina reads were produced for microbial eukaryotes and prokaryotes respectively. Adapters and primers were removed with Cutadapt v1.16 [73]. DADA2 v1.10.1 [74] was used for quality control, trimming, and inference of Operational Taxonomic Units (OTUs) as Amplicon Sequence Variants (ASVs). For both microbial eukaryotes and prokaryotes, the Maximum number of expected errors (MaxEE) was set to 2 and 4 for the forward and reverse reads respectively. No ambiguous bases (Ns) were allowed. Microbial eukaryotic sequences were trimmed to $220 \mathrm{bp}$ (forward) and $190 \mathrm{bp}$ (reverse), while prokaryotic sequences were trimmed to $225 \mathrm{bp}$ (both forward and reverse reads). A total of 28,876 and 19,604 OTUs were inferred for microbial eukaryotes and prokaryotes respectively.

OTUs were assigned taxonomy using the naïve Bayesian classifier method [75] together with the SILVA version 132 [76] database as implemented in DADA2. 
820 Eukaryotic OTUs were also BLASTed [77] against the Protist Ribosomal Reference

821 database $\left(\mathrm{PR}^{2}\right.$, version 4.10.0; [78]). When the taxonomic assignments for the

822 eukaryotes disagreed between SILVA and $\mathrm{PR}^{2}$, the conflict was resolved manually by

823 inspecting a pairwise alignment of the OTU and the closest hits from the two databases.

824 OTUs assigned to Metazoa, Streptophyta, nucleomorph, chloroplast, and mitochondria

825 were removed before further analysis. Archaea were removed from downstream

826 analyses as the used primers are not optimal for recovering this domain [79].

827 Each sample (corresponding to a specific gene, size fraction, and timepoint) was

828 subsampled with the rrarefy function from the R package Vegan [80] to 4,907 reads,

829 corresponding to the number of reads in the sample with the lowest sequencing depth,

830 to normalize for different sequencing depth between samples. OTUs present in $<10 \%$

831 of the samples were removed. After quality control and rarefaction, the number of

832 OTUs was 2,926 (1,561 bacteria, and 1,365 microeukaryotes; Table 1).

833 Due to a suboptimal sequencing of the amplicons, we did not use nanoplankton

834 samples of bacteria and protists from the period May 2010 to July 2012 (27 samples)

835 as well as March 2004 and February 2005. OTU read abundance for samples with

836 missing values were estimated using seasonally aware missing value imputation by

837 weighted moving average for time series as implemented in the R package imputeTS

$838[81]$.

839 Cell/particle dislodging or filter clogging during the sequential filtration process

840 may affect the taxonomic diversity observed in the different size fractions, with

841 nanoplankton DNA leaking into the picoplankton fraction, or picoplankton DNA

842 getting stuck in the nanoplankton fraction. To minimize the effects of cell/particle

843 dislodging or filter clogging on the diversity recovered from the different size fractions,

844 we calculated the sequence-abundance ratio for OTUs appearing in both pico- and 
nano-plankton fractions. When the ratio exceeded 2:1, we removed the OTU from the size fraction with the lowest number of reads. After subsampling and filtering the OTU

847 tables were joined for each time point, and since the samples had been normalized to

848 the same sequencing depth, we calculated the relative read abundance for the OTUs for

849 each year and aggregated over the corresponding months along the 10 years for the resident microbiota. This means that the relative abundance for both domains and size

851 fractions sums up to 1 for each month across ten years.

\section{Resident microbiota}

854 We defined ad hoc the resident microbiota as the set of OTUs present in $>30 \%$ of the samples over 10 years (that is, present in $>36$ months, not necessarily consecutive). residents included 355 eukaryotic and 354 bacteria OTUs (Table 1), and excluded a

859 substantial amount of rare OTUs, which can cause spurious correlations during network 860 construction due to sparsity [i.e. too many zeros] [22]. The relative abundance of the 861 taxonomic groups included in the resident microbiota was fairly stable from year to year (Figure 3).

Environmental variation and resident OTUs

865 All possible correlations among the measured environmental variables and resident 866 OTU richness and abundance were computed in $\mathrm{R}$ and plotted with the package 867 corrplot. Only significant Pearson correlation coefficients were considered $(\mathrm{p}<0.01)$, 868 and the p-values were corrected for multiple inference (Holm's method) using the 869 function rcorr.adjust from the $\mathrm{R}$ package RcmdrMisc. Unconstrained ordination 
analyses were carried out using NMDS based on Bray Curtis dissimilarities between

871 samples including resident OTUs only. Environmental variables were fitted to the

872 NMDS using the function envfit from the R package Vegan [80]. Only variables

873 displaying a significant correlation $(\mathrm{p}<0.05)$ were considered. Constrained ordination

874 was performed using distance-based redundancy analyses (dbRDA) in Vegan,

875 considering Bray Curtis dissimilarities between samples including resident OTUs only.

876 The most relevant variables for constrained ordination were selected by stepwise model

877 selection using 200 permutations, as implemented in ordistep (Vegan). Ordinations

878 were plotted using the R package ggplot2 and ggord. The amount of community

879 variance explained by the different environmental variables was calculated with Adonis

880 (Vegan) using 999 permutations. Resident OTUs displaying niche preference in terms

881 of Temperature and Daylength, the most important environmental variables, were

882 determined using the function niche.val from the R package EcolUtils with 1,000

883 permutations.

884

885 Delineation of seasons

886 Seasons were defined following Gasol et al. [36] with a small modification: months

887 with water temperature (at the sampling time) $>17^{\circ} \mathrm{C}$ and daylength $>14 \mathrm{~h} \mathrm{~d}^{-1}$ were

888 considered to be summer. Months with water temperature $<17{ }^{\circ} \mathrm{C}$ and $<11 \mathrm{~h} \mathrm{~d}^{-1}$ of

889 daylength were considered to be winter. Months with water temperature $>17^{\circ} \mathrm{C}$ and

890 daylength $<14 \mathrm{~h} \mathrm{~d}^{-1}$ were considered as autumn, while months with water temperature

$891<17^{\circ} \mathrm{C}$ and $>11 \mathrm{~h} \mathrm{~d}^{-1}$ of daylength were considered to be spring. The indicator value

892 [82] was calculated using the R package labdsv [83] to infer OTU seasonal preference.

894 Core microbiota delineated using networks 
895 The OTU table together with the 15 environmental variables were used to construct association networks using extended Local Similarity Analysis (eLSA) [84-86]. eLSA

897 was run on the OTU table with subsampled reads with default normalization: a z-score 898 transformation using the median and median absolute deviation. P-value estimations 899 were run under a mixed model that performs a random permutation test of a co900 occurrence only if the theoretical $p$-values for the comparison are $<0.05$. Bonferroni 901 false discovery rate (q) was calculated for all edges based on the p-values using the 902 p.adjust package in $\mathrm{R}$.

To detect environmentally-driven associations between OTUs induced by the

904 measured environmental variables we used the program EnDED [87].

905 Environmentally-driven associations indicate similar or different environmental 906 preferences between OTUs and not ecological interactions. In short, EnDED evaluates 907 associations between two OTUs that are both connected to the same environmental 908 variable based on a combination of four methods: Sign Pattern, Overlap, Interaction 909 Information, and Data Processing Inequality. These methods use the sign (positive or 910 negative) and the duration of the association, the relative abundance of OTUs as well 911 as environmental parameters to determine if an association is environmentally-driven. 912 If the four methods agreed that an association was environmentally-driven, then it was 913 removed from the network. The initial number of edges was 199,937, of which 180,345 914 were OTU-OTU edges that were at least in one triplet with an environmental parameter. 915 In total $65,280(\sim 33 \%)$ edges in the network were identified as indirect by EnDED and 916 removed. Afterward, only edges representing the strongest associations (i.e., absolute 917 local similarity score $|\mathrm{LS}|>0.7$, Spearman correlation $|\rho|>0.7, \mathrm{P}<0.001$ and $\mathrm{Q}<0.001$ ) 918 and nodes representing the resident OTUs were retained for downstream analysis and 919 are hereafter referred to as "core associations". Those OTUs participating in core 
920

921

922

923

924

925

926

associations were defined as core OTUs, although their involvement in ecological interactions need further experimental validation. Both core associations and core OTUs constitute the "core network", which also represents the core microbiota (both "core network" and "core microbiota" are used indistinctively). The core network was randomized using the Erdős-Rényi model [88], using 262 nodes and 1,411 edges.

For the core network, we calculated: 1) Density: quantifies the proportion of actual network connections out of the total number of possible connections, 2) Transitivity or Clustering coefficient: measures the probability that nodes connected to a node are also connected, forming tight clusters, 3) Average path length: mean number of steps (edges) along the shortest paths for all possible pairs of nodes in the network (a low average path length indicates that most species in the network are connected through a few intermediate species), 4) Degree: number of associations per node, 5) Betweenness centrality: measures how often an OTU (node) appears on the shortest paths between other OTUs in the network, 6) Closeness centrality: indicates how close a node is to all other nodes in a network, 7) Cliques: refers to sets of interconnected nodes where all possible connections are realized, 8) Modularity: measures the division of a given network into modules (that is, groups of OTUs that are highly interconnected between themselves).

The Degree, Betweenness centrality and Closeness centrality were used to identify central OTUs using ad hoc definitions. "Hub" OTUs were those with a score above the average for the three statistics and were normally among the top $25 \%$ in each score $[22,62,89]$. Specifically, hub OTUs featured a degree $>24$, Betweenness centrality $>0.03$ and Closeness centrality $>0.3$. Similarly, "connector" OTUs were defined as those featuring a relatively low degree and high centrality and could be seen as elements that connect different regions of a network or modules [50]. Connector 
945 OTUs featured a degree $<5$, Betweenness centrality $>0.03$ and Closeness centrality

$946>0.2$. Network statistics were calculated with igraph in R [90], Gephi [91] and

947 Cytoscape v3.6.1 [92]. Visualizations were made in Cytoscape v3.6.1. Modules in the 948 core network were identified with MCODE [93].

\section{DECLARATIONS}

952

Ethics approval and consent to participate

$954 \quad$ Not applicable

955

Consent for publication

957

Not applicable

958

960 DNA sequences and metadata are publicly available at the European Nucleotide

961 Archive (http://www.ebi.ac.uk/ena; accession numbers PRJEB23788 [18S rRNA genes] \& PRJEB38773 [16S rRNA genes]).

963

964 Competing interests

965 The authors declare that they have no competing interests

966

\section{$967 \quad$ Funding}

968 RL was supported by a Ramón y Cajal fellowship (RYC-2013-12554, MINECO, 969 Spain). IMD was supported by an ITN-SINGEK fellowship (ESR2-EU-H2020-MSCA- 
970 ITN-2015, Grant Agreement 675752 [ESR2] to RL). This work was supported by the

971 projects INTERACTOMICS (CTM2015-69936-P, MINECO, Spain to RL),

972 MicroEcoSystems (240904, RCN, Norway to RL), MINIME (PID2019-105775RB-

973 I00, AEI, Spain, to RL), ALLFLAGS (CTM2016-75083-R, MINECO to RM), MIAU

974 (RTI2018-101025-B-I00, to JMG) and DEVOTES (grant agreement $\mathrm{n}^{\circ} 308392$,

975 European Union to EG). It was further supported by Grup Consolidat de Recerca 976 2017SGR/1568 (Generalitat de Catalunya).

\section{Authors' contributions}

979 AKK \& RL designed the study. JMG, RM organized sampling. VB, CRG \& IF 980 collected samples, extracted the DNA, and organized its sequencing. AKK, RL \& ID 981 analyzed the data, while JMG, RM, IF, CRG \& EG, provided contextual ecological or 982 environmental pre-processed data. AKK, MFMB \& RL interpreted the results. AKK \& 983 RL wrote the manuscript. All authors contributed substantially to manuscript revisions. 984 All authors read and approved the final manuscript.

985

986

\section{Acknowledgements}

987 We thank all members of the Blanes Bay Microbial Observatory sampling and analyses 988 team. Bioinformatics analyses were performed at the MARBITS platform of the Institut 989 de Ciències del Mar (ICM; http://marbits.icm.csic.es). We thank the CSIC Open Access 990 Publication Support Initiative through the Unit of Information Resources for Research 991 (URICI) for helping to cover publication fees. 


\section{REFERENCES}

996 1. Gitay H, Wilson JB, Lee WG. Species Redundancy: A Redundant Concept? Journal of Ecology. 1996; 84(1):121-124.

2. Louca S, Parfrey LW, Doebeli M. Decoupling function and taxonomy in the global ocean microbiome. Science. 2016; 353:1272-1277.

3. Galand PE, Pereira O, Hochart C, Auguet JC, Debroas D. A strong link between marine microbial community composition and function challenges the idea of functional redundancy. ISME J. 2018; 12(10):2470-2478.

4. Shade A, Handelsman J. Beyond the Venn diagram: the hunt for a core microbiome. Environ Microbiol. 2012; 14(1):4-12.

5. Little AEF, Robinson CJ, Peterson SB, Raffa KF, Handelsman J. Rules of Engagement: Interspecies Interactions that Regulate Microbial Communities. Annual Review of Microbiology. 2008; 62(1):375-401.

6. Lennon JT, Jones SE. Microbial seed banks: the ecological and evolutionary implications of dormancy. Nat Rev Microbiol. 2011; 9(2):119-130.

7. Singer E, Wagner M, Woyke T. Capturing the genetic makeup of the active microbiome in situ. ISME J. 2017; 11(9):1949-1963.

8. Mestre M, Höfer J. The Microbial Conveyor Belt: Connecting the Globe through Dispersion and Dormancy. Trends Microbiol. 2021; in press.

9. Turnbaugh PJ, Ley RE, Hamady M, Fraser-Liggett CM, Knight R, Gordon JI. The Human Microbiome Project. Nature. 2007; 449(7164):804-810.

10. Wirth R, Kádár G, Kakuk B, Maróti G, Bagi Z, Szilágyi Á, Rákhely G, Horváth J, Kovács KL. The Planktonic Core Microbiome and Core Functions in the Cattle Rumen by Next Generation Sequencing. Front Microbiol. 2018; 9(2285).

11. Rubio-Portillo E, Kersting DK, Linares C, Ramos-Esplá AA, Antón J. Biogeographic Differences in the Microbiome and Pathobiome of the Coral Cladocora caespitosa in the Western Mediterranean Sea. Front Microbiol. 2018; 9(22).

12. Sweet MJ, Bulling MT. On the Importance of the Microbiome and Pathobiome in Coral Health and Disease. Frontiers in Marine Science. 2017; 4(9).

13. Lurgi M, Thomas T, Wemheuer B, Webster NS, Montoya JM. Modularity and predicted functions of the global sponge-microbiome network. Nature communications. 2019; 10(1):992.

14. Björk JR, O'Hara RB, Ribes M, Coma R, Montoya JM. The dynamic core microbiome: Structure, dynamics and stability. bioRxiv. 2018.

15. Delgado-Baquerizo M, Oliverio AM, Brewer TE, Benavent-González A, Eldridge DJ, Bardgett RD, Maestre FT, Singh BK, Fierer N. A global atlas of the dominant bacteria found in soil. Science. 2018; 359:320-325.

16. Logares R, Deutschmann IM, Junger PC, Giner CR, Krabberød AK, Schmidt TSB, Rubinat-Ripoll L, Mestre M, Salazar G, Ruiz-González C et al. Disentangling the mechanisms shaping the surface ocean microbiota. Microbiome. 2020; 8(1):55.

17. Gilbert JA, Steele JA, Caporaso JG, Steinbruck L, Reeder J, Temperton B, Huse S, McHardy AC, Knight R, Joint I et al. Defining seasonal marine microbial community dynamics. ISME J. 2012; 6(2):298-308.

18. Chow CE, Sachdeva R, Cram JA, Steele JA, Needham DM, Patel A, Parada AE, Fuhrman JA. Temporal variability and coherence of euphotic zone bacterial communities over a decade in the Southern California Bight. ISME J. 2013; 7(12):2259-2273. 
1044

1045

1046

1047

1048

1049

1050

1051

1052

1053

1054

1055

1056

1057

1058

1059

1060

1061

1062

1063

1064

1065

1066

1067

1068

1069

1070

1071

1072

1073

1074

1075

1076

1077

1078

1079

1080

1081

1082

1083

1084

1085

1086

1087

1088

1089

1090

1091

1092

19. Worden AZ, Follows MJ, Giovannoni SJ, Wilken S, Zimmerman AE, Keeling PJ. Rethinking the marine carbon cycle: factoring in the multifarious lifestyles of microbes. Science. 2015; 347(6223):1257594.

20. Krabberød AK, Bjorbækmo MFM, Shalchian-Tabrizi K, Logares R. Exploring the oceanic microeukaryotic interactome with metaomics approaches. Aquat Microb Ecol. 2017; 79(1):1-12.

21. Bjorbaekmo MFM, Evenstad A, Rosaeg LL, Krabberod AK, Logares R. The planktonic protist interactome: where do we stand after a century of research? ISME J. 2020; 14(2):544-559.

22. Röttjers L, Faust K. From hairballs to hypotheses - biological insights from microbial networks. FEMS Microbiol Rev. 2018; 42(6):761-780.

23. Chow CE, Kim DY, Sachdeva R, Caron DA, Fuhrman JA. Top-down controls on bacterial community structure: microbial network analysis of bacteria, T4like viruses and protists. ISME J. 2014.

24. Layeghifard M, Hwang DM, Guttman DS. Disentangling Interactions in the Microbiome: A Network Perspective. Trends Microbiol. 2017; 25(3):217-228.

25. Fuhrman JA, Cram JA, Needham DM. Marine microbial community dynamics and their ecological interpretation. Nat Rev Microbiol. 2015; 13(3):133-146.

26. Lima-Mendez G, Faust K, Henry N, Decelle J, Colin S, Carcillo F, Chaffron S, Ignacio-Espinosa JC, Roux S, Vincent $\mathrm{F}$ et al. Determinants of community structure in the global plankton interactome. Science. 2015; 348(6237):1262073.

27. Ponisio LC, Valdovinos FS, Allhoff KT, Gaiarsa MP, Barner A, Guimarães PR, Hembry DH, Morrison B, Gillespie R. A Network Perspective for Community Assembly. Frontiers in Ecology and Evolution. 2019; 7(103).

28. Chaffron S, Rehrauer H, Pernthaler J, von Mering C. A global network of coexisting microbes from environmental and whole-genome sequence data. Genome Res. 2010; 20(7):947-959.

29. Krabberod AK, Bjorbaekmo MFM, Shalchian-Tabrizi K, Logares R. Exploring the oceanic microeukaryotic interactome with metaomics approaches. Aquat Microb Ecol. 2017; 79(1):1-12.

30. Cram JA, Xia LC, Needham DM, Sachdeva R, Sun F, Fuhrman JA. Cross-depth analysis of marine bacterial networks suggests downward propagation of temporal changes. ISME J. 2015; 9(12):2573-2586.

31. Steele JA, Countway PD, Xia L, Vigil PD, Beman JM, Kim DY, Chow CE, Sachdeva R, Jones AC, Schwalbach MS et al. Marine bacterial, archaeal and protistan association networks reveal ecological linkages. ISME J. 2011; 5(9):1414-1425.

32. Needham DM, Fuhrman JA. Pronounced daily succession of phytoplankton, archaea and bacteria following a spring bloom. Nat Microbiol. 2016; 1(4): 16005 .

33. Deutschmann IM, Lima-Mendez G, Krabberød AK, Raes J, Vallina SM, Faust $\mathrm{K}$, Logares R. Disentangling environmental effects in microbial association networks. preprint. 2021.

34. Giner CR, Balague V, Krabberod AK, Ferrera I, Rene A, Garces E, Gasol JM, Logares R, Massana R. Quantifying long-term recurrence in planktonic microbial eukaryotes. Mol Ecol. 2019; 28(5):923-935.

35. Alonso-Saez L, Balague V, Sa EL, Sanchez O, Gonzalez JM, Pinhassi J, Massana R, Pernthaler J, Pedros-Alio C, Gasol JM. Seasonality in bacterial 
diversity in north-west Mediterranean coastal waters: assessment through clone libraries, fingerprinting and FISH. FEMS Microbiol Ecol. 2007; 60(1):98-112.

36. Gasol JM, Cardelus C, Moran XAG, Balague V, Forn I, Marrase C, Massana R, Pedros-Alio C, Sala MM, Simo R et al. Seasonal patterns in phytoplankton photosynthetic parameters and primary production at a coastal NW Mediterranean site. Sci Mar. 2016; 80(S1):63-77.

37. Watts DJ, Strogatz SH. Collective dynamics of 'small-world' networks. Nature. 1998; 393(6684):440-442.

38. Pedrós-Alió C. The rare bacterial biosphere. Ann Rev Mar Sci. 2012; 4:449466.

39. Mestre M, Höfer J, Sala MM, Gasol JM. Seasonal Variation of Bacterial Diversity Along the Marine Particulate Matter Continuum. Front Microbiol. 2020; 11(1590).

40. Auladell A, Sánchez P, Sánchez O, Gasol JM, Ferrera I. Long-term seasonal and interannual variability of marine aerobic anoxygenic photoheterotrophic bacteria. ISME J. 2019; 13(8):1975-1987.

41. Berdjeb L, Parada A, Needham DM, Fuhrman JA. Short-term dynamics and interactions of marine protist communities during the spring-summer transition. ISME J. 2018; 12:1907-1917.

42. Lambert S, Tragin M, Lozano JC, Ghiglione JF, Vaulot D, Bouget FY, Galand PE. Rhythmicity of coastal marine picoeukaryotes, bacteria and archaea despite irregular environmental perturbations. ISME J. 2019; 13(2):388-401.

43. Charles F, Lantoine F, Brugel S, Chrétiennot-Dinet M-J, Quiroga I, Rivière B. Seasonal survey of the phytoplankton biomass, composition and production in a littoral NW Mediterranean site, with special emphasis on the picoplanktonic contribution. Estuarine, Coastal and Shelf Science. 2005; 65(1):199-212.

44. Milici M, Deng Z-L, Tomasch J, Decelle J, Wos-Oxley ML, Wang H, Jáuregui R, Plumeier I, Giebel H-A, Badewien TH et al. Co-occurrence Analysis of Microbial Taxa in the Atlantic Ocean Reveals High Connectivity in the FreeLiving Bacterioplankton. Front Microbiol. 2016; 7(649).

45. Newman M. Networks: Oxford University Press; 2018.

46. Coyte KZ, Schluter J, Foster KR. The ecology of the microbiome: Networks, competition, and stability. Science. 2015; 350:663-666.

47. Vellend M. The theory of ecological communities. Princeton: Princeton University Press; 2016.

48. Stouffer DB, Bascompte J. Compartmentalization increases food-web persistence. Proceedings of the National Academy of Sciences. 2011; 108:36483652.

49. Krause AE, Frank KA, Mason DM, Ulanowicz RE, Taylor WW. Compartments revealed in food-web structure. Nature. 2003; 426(6964):282-285.

50. Olesen JM, Bascompte J, Dupont YL, Jordano P. The modularity of pollination networks. Proceedings of the National Academy of Sciences. 2007; 104:1989119896.

51. Medeiros LP, Garcia G, Thompson JN, Guimarães PR. The geographic mosaic of coevolution in mutualistic networks. Proceedings of the National Academy of Sciences. 2018; 115:12017-12022.

52. Tripp HJ, Schwalbach MS, Meyer MM, Kitner JB, Breaker RR, Giovannoni SJ. Unique glycine-activated riboswitch linked to glycine-serine auxotrophy in SAR11. Environ Microbiol. 2009; 11(1):230-238. 
53. Carini P, Steindler L, Beszteri S, Giovannoni SJ. Nutrient requirements for growth of the extreme oligotroph 'Candidatus Pelagibacter ubique' HTCC1062 on a defined medium. ISME J. 2013; 7(3):592-602.

54. Carini P, Campbell EO, Morré J, Sañudo-Wilhelmy SA, Cameron Thrash J, Bennett SE, Temperton B, Begley T, Giovannoni SJ. Discovery of a SAR11 growth requirement for thiamin's pyrimidine precursor and its distribution in the Sargasso Sea. ISME J. 2014; 8(8):1727-1738.

55. Włodarczyk A, Selão TT, Norling B, Nixon PJ. Newly discovered Synechococcus sp. PCC 11901 is a robust cyanobacterial strain for high biomass production. Communications Biology. 2020; 3(1):215.

56. Becker JW, Hogle SL, Rosendo K, Chisholm SW. Co-culture and biogeography of Prochlorococcus and SAR11. ISME J. 2019; 13(6):1506-1519.

57. Chafee $M$, Fernàndez-Guerra $A$, Buttigieg PL, Gerdts G, Eren AM, Teeling H, Amann RI. Recurrent patterns of microdiversity in a temperate coastal marine environment. ISME J. 2018; 12(1):237-252.

58. Paine RT. A Note on Trophic Complexity and Community Stability. The American Naturalist. 1969; 103(929):91-93.

59. Banerjee S, Schlaeppi K, van der Heijden MGA. Keystone taxa as drivers of microbiome structure and functioning. Nat Rev Microbiol. 2018; 16:567-576.

60. Berry D, Widder S. Deciphering microbial interactions and detecting keystone species with co-occurrence networks. Front Microbiol. 2014; 5(MAY):219.

61. Freilich MA, Wieters E, Broitman BR, Marquet PA, Navarrete SA. Species cooccurrence networks: Can they reveal trophic and non-trophic interactions in ecological communities? Ecology. 2018; 99(3):690-699.

62. Banerjee S, Kirkby CA, Schmutter D, Bissett A, Kirkegaard JA, Richardson AE. Network analysis reveals functional redundancy and keystone taxa amongst bacterial and fungal communities during organic matter decomposition in an arable soil. Soil Biology and Biochemistry. 2016; 97:188-198.

63. Pester M, Bittner N, Deevong P, Wagner M, Loy A. A 'rare biosphere' microorganism contributes to sulfate reduction in a peatland. ISME J. 2010; 4(12):1591-1602.

64. Zhu F, Massana R, Not F, Marie D, Vaulot D. Mapping of picoeucaryotes in marine ecosystems with quantitative PCR of the 18S rRNA gene. FEMS Microbiol Ecol. 2005; 52(1):79-92.

65. Seymour JR, Amin SA, Raina JB, Stocker R. Zooming in on the phycosphere: the ecological interface for phytoplankton-bacteria relationships. Nat Microbiol. 2017; 2:17065.

66. Farnelid HM, Turk-Kubo KA, Zehr JP. Identification of Associations between Bacterioplankton and Photosynthetic Picoeukaryotes in Coastal Waters. Front Microbiol. 2016; 7(339).

67. Auladell A, Barberán A, Logares R, Garcés E, Gasol JM, Ferrera I. Seasonal niche differentiation between evolutionary closely related marine bacteria. bioRxiv. 2020.

68. Grasshoff K, Kremling K, Ehrhardt M. Methods of Seawater Analysis: Third, Completely Revised and Extended Edition; 2007.

69. Massana R, Castresana J, Balague V, Guillou L, Romari K, Groisillier A, Valentin K, Pedros-Alio C. Phylogenetic and ecological analysis of novel marine stramenopiles. Appl Environ Microbiol. 2004; 70(6):3528-3534.

70. Stoeck T, Bass D, Nebel M, Christen R, Jones MD, Breiner HW, Richards TA. Multiple marker parallel tag environmental DNA sequencing reveals a highly 
complex eukaryotic community in marine anoxic water. Mol Ecol. 2010; 19 Suppl 1(SUPPL. 1):21-31.

71. Herlemann DPR, Labrenz M, Jürgens K, Bertilsson S, Waniek JJ, Andersson AF. Transitions in bacterial communities along the $2000 \mathrm{~km}$ salinity gradient of the Baltic Sea. ISME J. 2011; 5(10):1571-1579.

72. Apprill A, McNally S, Parsons R, Weber L. Minor revision to V4 region SSU rRNA 806R gene primer greatly increases detection of SAR11 bacterioplankton. Aquat Microb Ecol. 2015; 75(2):129-137.

73. Martin M. Cutadapt removes adapter sequences from high-throughput sequencing reads. EMBnet Journal. 2011; 17(1):10-12.

74. Callahan BJ, McMurdie PJ, Rosen MJ, Han AW, Johnson AJ, Holmes SP. DADA2: High-resolution sample inference from Illumina amplicon data. Nat Methods. 2016; 13(7):581-583.

75. Wang Q, Garrity GM, Tiedje JM, Cole JR. Naive Bayesian classifier for rapid assignment of rRNA sequences into the new bacterial taxonomy. Appl Environ Microbiol. 2007; 73(16):5261-5267.

76. Quast C, Pruesse E, Yilmaz P, Gerken J, Schweer T, Yarza P, Peplies J, Glockner FO. The SILVA ribosomal RNA gene database project: improved data processing and web-based tools. Nucleic Acids Res. 2013; 41(Database issue):D590-596.

77. Altschul SF, Gish W, Miller W, Myers EW, Lipman DJ. Basic local alignment search tool. Journal of molecular biology. 1990; 215(3):403-410.

78. Guillou L, Bachar D, Audic S, Bass D, Berney C, Bittner L, Boutte C, Burgaud $\mathrm{G}$, de Vargas $\mathrm{C}$, Decelle $\mathrm{J}$ et al. The Protist Ribosomal Reference database (PR2): a catalog of unicellular eukaryote small sub-unit rRNA sequences with curated taxonomy. Nucleic Acids Res. 2013; 41(Database issue):D597-604.

79. McNichol J, Berube PM, Biller SJ, Fuhrman JA. Evaluating and Improving SSU rRNA PCR Primer Coverage via Metagenomes from Global Ocean Surveys. bioRxiv. 2020.

80. Oksanen J, Guillaume Blanchet FFM, Kindt R, Legendre P, McGlinn D, Minchin PR, O'Hara RB, Simpson GL, Solymos P, Stevens MHH et al. vegan: Community Ecology Package. R package. In.; 2016.

81. Moritz S. imputeTS: Time Series Missing Value Imputation. In.; 2017.

82. Dufrêne M, Legendre P. Species assemblages and indicator species: The need for a flexible asymmetrical approach. Ecological Monographs. 1997.

83. Roberts DW. labdsv: Ordination and Multivariate Analysis for Ecology. R package version 1.8-0. In.; 2016.

84. Ruan Q, Dutta D, Schwalbach MS, Steele JA, Fuhrman JA, Sun F. Local similarity analysis reveals unique associations among marine bacterioplankton species and environmental factors. Bioinformatics. 2006; 22(20):2532-2538.

85. Xia LC, Ai D, Cram JA, Liang X, Fuhrman JA, Sun F. Statistical significance approximation in local trend analysis of high-throughput time-series data using the theory of Markov chains. BMC Bioinformatics. 2015; 16(1):301.

86. Xia LC, Ai D, Cram J, Fuhrman JA, Sun F. Efficient statistical significance approximation for local similarity analysis of high-throughput time series data. Bioinformatics. 2013; 29(2):230-237.

87. Deutschmann IM, Lima-Mendez G, Krabberød AK, Raes J, Vallina SM, Faust $\mathrm{K}$, Logares R. Disentangling environmental effects in microbial association networks. ResearchSquare. 2020. 
1241 88. Erdős P, Rényi A. On random graphs. Publicationes Mathematicae. 1959;

89. Banerjee S, Baah-Acheamfour M, Carlyle CN, Bissett A, Richardson AE, Siddique T, Bork EW, Chang SX. Determinants of bacterial communities in Canadian agroforestry systems. Environ Microbiol. 2016; 18(6):1805-1816.

90. Yu G, Chen Y-s, Guo Y-c. Design of integrated system for heterogeneous network query terminal. Journal of Computer Applications. 2009; 29(8):21912193.

91. Bastian M, Heymann S, Jacomy M. Gephi: an open source software for exploring and manipulating networks. In: International AAAI Conference on Weblogs and Social Media. 2009.

92. Smoot ME, Ono K, Ruscheinski J, Wang PL, Ideker T. Cytoscape 2.8: New features for data integration and network visualization. Bioinformatics. 2011.

93. Bader GD, Hogue CW. An automated method for finding molecular complexes in large protein interaction networks. BMC Bioinformatics. 2003; 4(1):2. 


\section{FIGURE LEGENDS}

\section{Figure 1. The Blanes Bay Microbial Observatory and the variation of its resident} microbiota and measured environmental variables over ten years. A) Location of the Blanes Bay Microbial Observatory. B) All possible correlations between the measured environmental variables including the richness and abundance of resident OTUs (NB: only 709 resident OTUs are considered, see Table1). Only significant Pearson correlation coefficients are shown $(\mathrm{p}<0.01)$. The $\mathrm{p}$-values were corrected for multiple inference (Holm's method). C) Unconstrained ordination (NMDS based on Bray Curtis dissimilarities) of communities including resident OTUs only, to which environmental variables were fitted. Only variables with a significant fit are shown $(\mathrm{P}<0.05)$. Arrows indicate the direction of the gradient and their length represents the strength of the correlation between resident OTUs and a particular environmental variable. The color of the samples (circles) indicates the season to which they belong. The bottom-left arrow indicates the direction of the seasonal change. PNF $=$ photosynthetic nanoflagellates. D) Constrained ordination (Distance-based redundancy analyses, dbRDA, using Bray Curtis dissimilarities) including only the most relevant variables after stepwise model selection using permutation tests. Each axis (i.e., dbRDA1 and dbRDA2) indicates the amount of variance it explains according to the associated eigenvalues. The color of the samples (circles) indicates the season to which they belong. Arrows indicate the direction of the gradient and their length represents the strength of the correlation between resident OTUs and a particular environmental variable. The bottom-left arrow indicates the direction of the seasonal change. E-F) Resident OTUs displaying different niche preferences (blueish areas) in terms of the two most important abiotic variables: Temperature E) and Daylength F). The red dots 
indicate the randomization mean, and the orange curves represent the confidence limits.

1285 Black dots indicate individual OTUs for which temperature or daylength preferences

1286 are significantly $(\mathrm{p}<0.05)$ higher or lower than a random distribution over 10 years. At

1287 least two assemblages with different niches become evident: one preferring higher

1288 temperature and longer days (summer/spring), and another one preferring lower

1289 temperature and shorter days (winter/autumn). Note that several OTUs associated to

1290 Spring or Autumn are not expected to be detected with this approach, as their preferred

1291 temperature or daylength may not differ significantly from the randomized mean.

Figure 2. Core microbiota resulting from 10 years of monthly pico- and

nanoplankton relative abundances. A) Core network including bacteria and microbial eukaryotic OTUs that occur $\geq 30 \%$ of the time during the studied decade (i.e.

$\mathrm{Q}<0.001$, absolute local similarity score $|\mathrm{LS}|>0.7$, Spearman correlation $|\rho|>0.7)$,

1298 where detected environmentally-driven edges were removed. The color of the edges

1299 (links) indicates whether the association is positive (grey) or negative (red). The shape

1300 of nodes indicates bacteria (rhomboid) or microbial eukaryotes (circle), and the color

1301 of nodes represents species seasonal preferences, determined using the indicator value

1302 (indval, $\mathrm{p}<0.05$ ). Node size indicates OTU relative abundance. B) Core network as a

1303 Circos plot, indicating the high-rank taxonomy of the core OTUs. Since $95 \%$ of the

1304 associations are positive (see Table 2), we do not indicate whether an edge is positive 1305 or negative.

1307 Figure 3. The monthly variation in the resident and core microbiotas over 10

1308 years. Upper panels: The resident microbiota is defined as those eukaryotes and 
bacteria that occur in at least $30 \%$ of the samples over 10 years. The relative OTU

1310 abundance (left panel) and number of OTUs (right panel) for different domains and

1311 taxonomic levels in the resident microbiota are shown. Note that the relative abundance

1312 of Bacteria vs. Eukaryotes does not necessarily reflect organismal abundances on the

1313 sampling site, but the amplicon relative abundance after PCR. Relative abundances

1314 were calculated for each year and aggregated over the corresponding months along the

131510 years for the resident microbiota, then split into size fractions (NB: relative

1316 abundance for both domains and size fraction sums up to 1 for each month across ten

1317 years). Lower panels: Core microbiota over 10 years. The relative abundances of core

1318 OTUs reflect the remaining proportions after removing all the OTUs that were not 1319 strongly associated when building networks. Relative OTU abundance (left panel) and 1320 number of OTUs (right panel) for different domains and taxonomic levels among the 1321 core OTUs.

1323 Figure 4. Pico- and nanoplankton core sub-networks. The shape of the nodes

1324 indicates bacteria (rhomboid) or microbial eukaryotes (circle), and the color of nodes 1325 represents species seasonal preferences, determined using the indicator value $(\mathrm{p}<0.05)$.

1326 The color of the edges indicates if the association is positive (grey) or negative (red).

1327 Node size indicates OTU relative abundance from the core microbiota.

1329 Figure 5. Main modules in the core network. Modules with MCODE score $>4$ are 1330 shown for picoplankton (upper panel) and nanoplankton (lower panel). For each 1331 module, the MCODE score and relative amplicon abundance of the taxa included in it 1332 (as \% of the resident microbiota) are indicated. In addition, the numbers of edges and 1333 OTUs within the modules are shown as edges/OTUs; this quotient estimates the average 
1334 number of edges per OTU within the different modules. The edges represent 1335 correlations with $|\mathrm{LS}|>0.7,|\rho|>0.7, \mathrm{P}<0.001$ and $\mathrm{Q}<0.001$. The color of the edges 1336 indicates positive (grey) or negative (red) associations. The shape of nodes indicates 1337 bacteria (rhomboid) or microbial eukaryotes (circle), and the color of nodes represents 1338 species seasonal preferences, determined using the indicator value $(\mathrm{p}<0.05)$. $\mathrm{pb}=$ 1339 Proteobacteria

\section{TABLE TITLES}

1344 Table 1. Description of the datasets.

1345 Table 2. Core associations. See Figure 2.

1346 Table 3. Core network and sub-networks statistics.

1347 Table 4. Core associations within and between taxonomic domains and size fractions.

1348 Table 5: Subnetworks including core OTUs displaying seasonal preference.

1349 Table 6. Central OTUs.

\section{ADDITIONAL FILES}

\section{Additional file 1: Table S1}

1355 Relative abundance of bacterial and protistan lineages that are part of the resident and 1356 core microbiotas. 
1359

1360

1361

1362

1363

1364

1365

1366

1367

1368

1369

1370

1371

1372

1373

1374

1375

1376

1377

1378

1379

1380

1381

1382

1383

Additional file 1: Table S2

Relative abundance of core bacterial taxa.

Additional file 1: Table S3

Relative abundance of core eukaryotic taxa.

\section{Additional file 1: Table S4}

Indicator value for core OTUs in the picoplankton. Sorted by season/kingdom and relative amplicon abundance.

\section{Additional file 1: Table S5}

Indicator value for core OTUs in the nanoplankton. Sorted by season/ kingdom and relative amplicon abundance.

\section{Additional file 1: Table S6}

Core OTUs without seasonal preference.

\section{Additional file 1: Table S7}

Module description.

\section{Additional file 1: Table S8}

OTUs within modules. 
Additional file 2: Figure S1

1385 Panel A shows the full network constructed with the resident microbiota (that is, OTUs

1386 present in $>30 \%$ of the samples over 10 years; Table 1). Panel B displays network

1387 elements that were removed as they did not fulfill the cut-offs (that is, highly significant

1388 correlations ( $\mathrm{P} \& \mathrm{Q}<0.001$ ), local similarity scores $>|0.7|$ and Spearman correlations

$1389>|0.7|)$.

1390

$1391 \quad$ Additional file 3: Figure S2

1392 OTU relative abundance vs. degree shows no relationship in the core network. 
bioRxiv preprint doi: https://doi.org/10.1101/2021.03.18.435965; this version posted June 24, 2021. The copyright holder for this preprint (which was not certified by peer review) is the author/funder, who has granted bioRxiv a license to display the preprint in perpetuity. It is made available under aCC-BY-NC-ND 4.0 International license.

\section{Supplementary Figures}

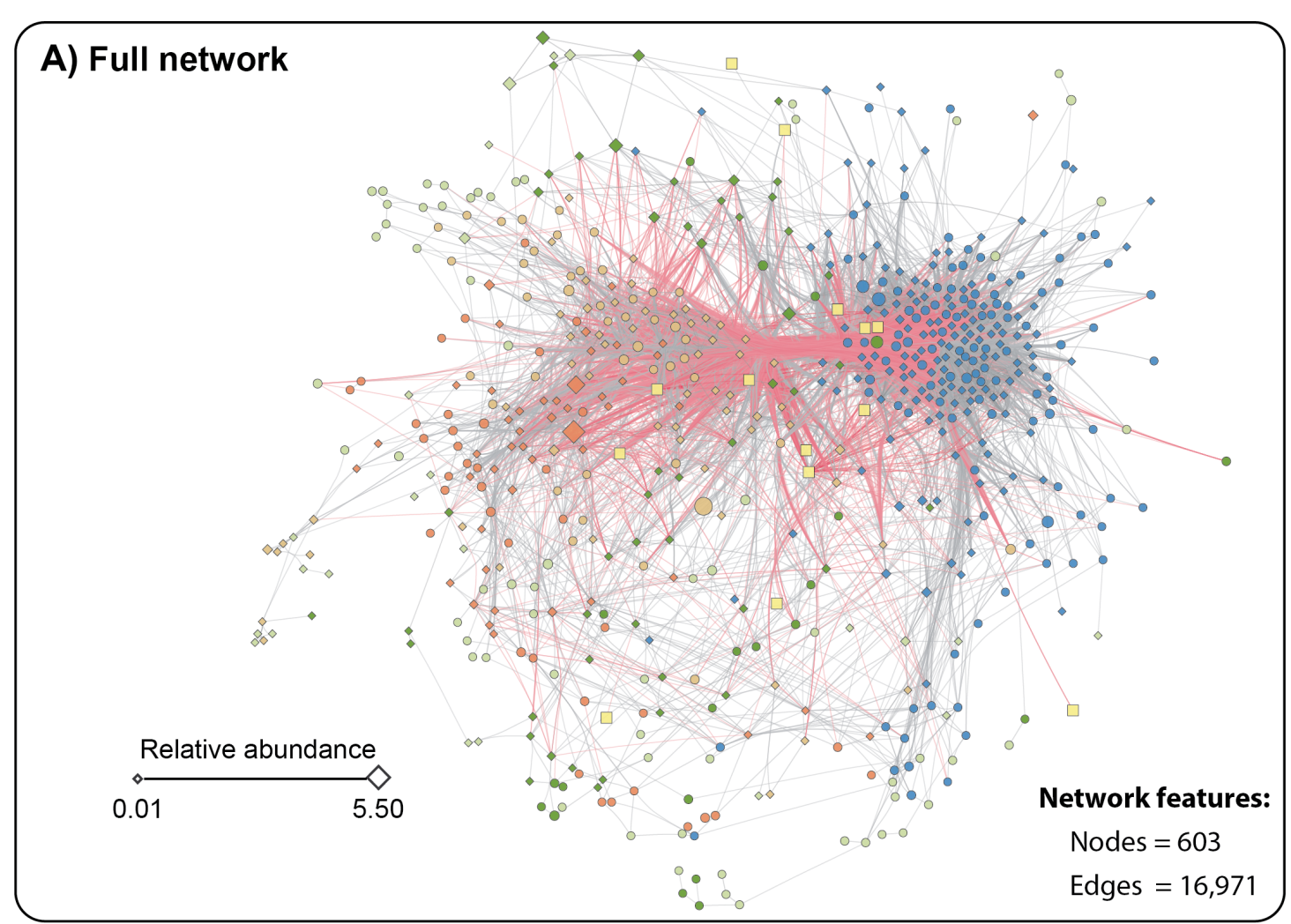

Figure S1. Panel A shows the full network constructed with the resident microbiota (that is, OTUs present in $>30 \%$ of the samples over 10 years; Table 1). Panel B displays network elements that were removed as they did not fulfill the cut-offs (that is, highly significant correlations ( $\mathrm{P} \& \mathrm{Q}<0.001$ ), local similarity scores $>|0.7|$ and Spearman correlations $>|0.7|)$.

B) Removed elements

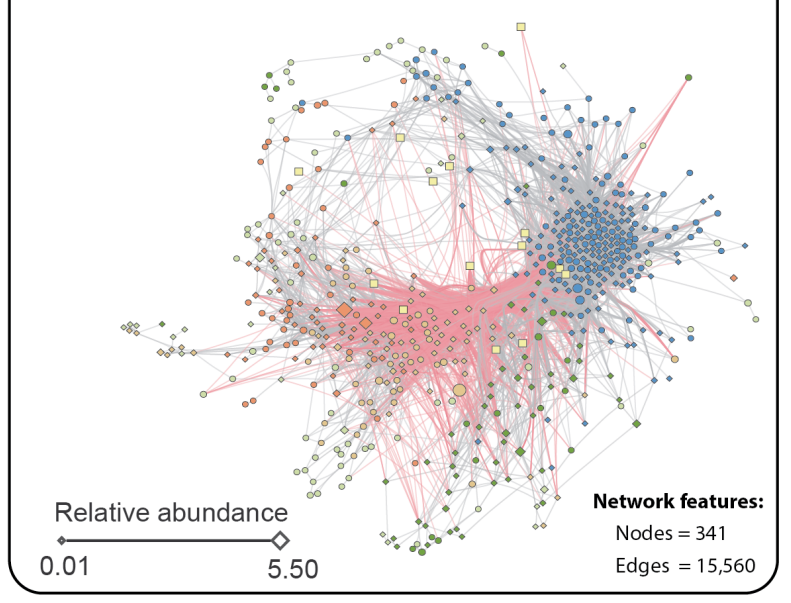




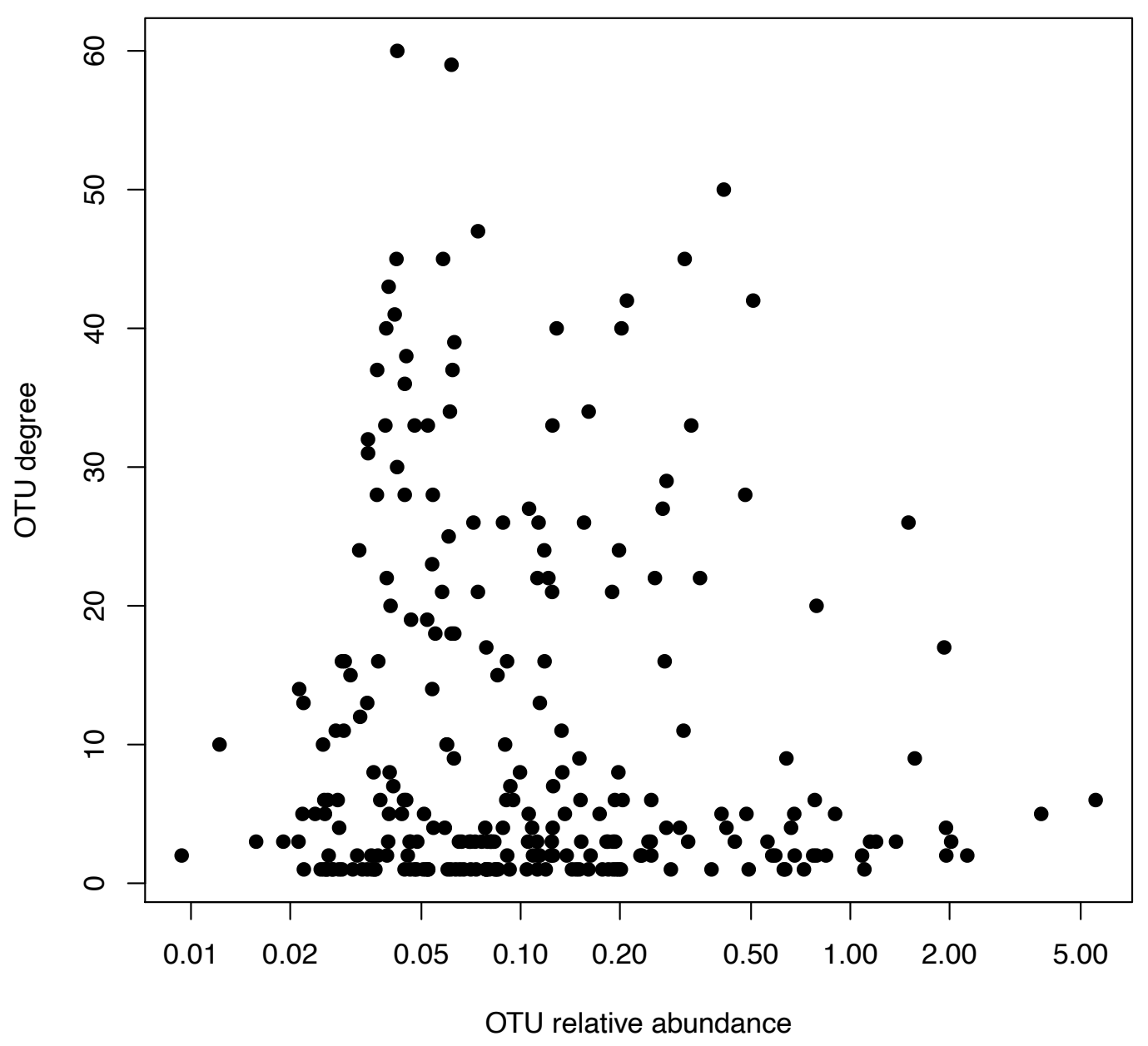

1410 Figure S2. OTU relative abundance vs. degree shows no relationship in the core 